\title{
Huntington's Disease Patient-Derived Astrocytes Display Electrophysiological Impairments and Reduced Neuronal Support
}

\author{
Veronica J. Garcia't, David J. Rushton ${ }^{1,2+}$, Colton M. Tom ${ }^{1}$, Nicholas D. Allen², \\ Paul J. Kemp ${ }^{2}$, Clive N. Svendsen ${ }^{1}$ and Virginia B. Mattis ${ }^{1 *}$ \\ ${ }^{1}$ Board of Governors Regenerative Medicine Institute, Cedars-Sinai Medical Center, Los Angeles, CA, United States, \\ ${ }^{2}$ Divisions of Biomedicine and Neuroscience, School of Biosciences, Cardiff University, Cardiff, United Kingdom
}

OPEN ACCESS

Edited by:

Alexei Verkhratsky,

The University of Manchester,

United Kingdom

Reviewed by:

Augustas Pivoriūnas,

State Research Institute Centre for Innovative Medicine, Lithuania

Rheinallt Parri,

Aston University, United Kingdom

*Correspondence:

Virginia B. Mattis

VirginiaMattis@gmail.com

${ }^{\dagger}$ These authors have contributed equally to this work

Specialty section:

This article was submitted to

Neurodegeneration,

a section of the journal

Frontiers in Neuroscience

Received: 27 March 2019

Accepted: 11 June 2019

Published: 28 June 2019

Citation: Garcia VJ, Rushton DJ, Tom CM, Allen ND, Kemp PJ, Svendsen CN and Mattis VB (2019) Huntington's Disease Patient-Derived Astrocytes Display Electrophysiological Impairments and Reduced Neuronal

Support. Front. Neurosci. 13:669.

doi: 10.3389/fnins.2019.00669
In Huntington's disease (HD), while the ubiquitously expressed mutant Huntingtin (mtHTT) protein primarily compromises striatal and cortical neurons, glia also undergo disease-contributing alterations. Existing HD models using human induced pluripotent stem cells (iPSCs) have not extensively characterized the role of $\mathrm{mHTT}$ in patientderived astrocytes. Here physiologically mature astrocytes are generated from HD patient iPSCs. These human astrocytes exhibit hallmark HD phenotypes that occur in mouse models, including impaired inward rectifying $\mathrm{K}^{+}$currents, lengthened spontaneous $\mathrm{Ca}^{2+}$ waves and reduced cell membrane capacitance. HD astrocytes in co-culture provided reduced support for the maturation of iPSC-derived neurons. In addition, neurons exposed to chronic glutamate stimulation are not protected by HD astrocytes. This iPSC-based HD model demonstrates the critical effects of $\mathrm{mtHTT}$ on human astrocytes, which not only broadens the understanding of disease susceptibility beyond cortical and striatal neurons but also increases potential drug targets.

Keywords: Huntington's disease, astrocyte, neuron, iPSC, co-culture, neurodegeneration, HTT, HD

\section{INTRODUCTION}

Huntington's disease (HD) is a fatal neurodegenerative disease that presents with involuntary motor control as well as cognitive and psychiatric disturbances. HD is directly linked to over 40 CAG trinucleotide repeats in the first exon of the Huntingtin gene (Andrew et al., 1993). The number of CAG repeats has a positive correlation between the age-of-onset of symptoms and severity of the disease. When translated, the CAG repeat produces an expanded polyglutamine repeat (polyQ) in the mutant Huntingtin (mtHTT) protein. Though mtHTT is ubiquitously expressed, it primarily leads to dysfunction and progressive death of medium spiny neurons (MSNs) in the striatum, and subsequently cortical projection neurons (Macdonald and Halliday, 2002; Thu et al., 2010; Ross and Tabrizi, 2011). It is still unclear whether this expansion causes a gain of toxic functions, loss of physiological functions of the protein, or both (see Jimenez-Sanchez et al., 2017 for review). Despite knowing for over two decades that expanded HTT underlies HD, there is still no successful treatment or comprehensive understanding of the selective neuronal loss. 
While neuronal degeneration is central to $\mathrm{HD}$, glial cells also display disease-related phenotypes in HD patients and rodent models (Khakh et al., 2017). Specifically, dysfunctional astrocytes have been shown to play critical roles in the neuronal decline (Khakh and Sofroniew, 2014; Tong et al., 2014; Jiang et al., 2016). HD rodent astrocytes demonstrate impaired glutamate uptake, which increases activation of neuronal NMDA receptors leading to neurotoxicity (Milnerwood et al., 2010). In addition, impaired $\mathrm{K}^{+}$buffering via reduced inward rectifying $\mathrm{K}^{+}$currents causes rapid changes to the excitability of neurons in response to rates of action potential propagation (Tong et al., 2014).

Induced pluripotent stem cells (iPSCs) (Takahashi and Yamanaka, 2006) can be differentiated to provide a disease-relevant source of human neural cell types. Human iPSC-derived HD neurons have been co-cultured with healthy rodent-derived glia, which improve the electrophysiological function and survival of iPSC-derived neurons (Tang et al., 2013). However, the rodent origin prohibits the study of human HD glial phenotypes. Patient-derived iPSCs are a promising cell source to derive mature astrocytes to model their dysfunction in neurodegenerative diseases (Khakh et al., 2017). Co-culture with neurons may enhance the astrocyte maturation process; however, pure astrocytes would be ideal to distinguish the specific role of astrocytes versus neurons in HD. Furthermore, deriving terminally differentiated astrocytes without passaging would reduce the risk of generating reactive astrocytes that could confound disease-specific phenotypes.

In a chimeric mouse model, human HD iPSC-derived glia surrounding non-diseased murine MSNs led to neuronal hyperexcitability and reduced motor performance (Benraiss et al., 2016). In addition, HD patient iPSC-derived astrocytes display autophagocytic vacuoles (Juopperi et al., 2012) and increased evoked inflammatory responses (Hsiao et al., 2014). However, these studies did not assess the functional maturity of the iPSC-derived astrocytes. And, notably, studies to date have yet to investigate whether patient-derived HD astrocytes directly affect human neuronal function.

Here, we present a novel protocol to differentiate patient iPSCs into a pure population of human HD astrocytes. The astrocytes were functionally mature, displaying electrophysiological profiles not typically observed in less mature or stress-activated astrocytes. This included inward rectifying $\mathrm{K}^{+}$currents that were altered in the HD astrocytes, which corroborates previous results in HD (Tong et al., 2014). At early stages in co-culture, HD astrocytes, compared to control astrocytes, provided less support for the maturation of $\mathrm{HD}$ and control neurons. Furthermore, the HD astrocytes, compared to controls, did not protect $\mathrm{HD}$ or control neurons from toxicity following chronic glutamate exposure. Our data from iPSC-based HD astrocytes recapitulates findings from patient post-mortem samples and HD mouse astrocytes (Khakh et al., 2017), validating this novel use of patient iPSCs to derive pure functional astrocytes. These human astrocytes provide an ideal model to investigate mechanisms of astrocyte-neuron interactions in HD as well as to identify novel therapeutic targets.

\section{MATERIALS AND METHODS}

\section{iPSC Lines}

The iPSC lines used in this study were purchased from the Induced Pluripotent Stem Cell Core at Cedars-Sinai (Los Angeles, CA, United States). All lines were reprogramed from fibroblast cells. Control lines included CS83iCTR33n (female, age 21, clinically normal); CS00iCTR21n (male, age 6, clinically normal); and CS25iCTR18n (male, 76, clinically normal, brother of affected sibling). HD lines include CS09iHD109n (female, age unknown, clinically affected with 109 and 119 CAG repeats) and CS77iHD77n (male, age unknown, clinically affected with 77 CAG repeats). These lines have tested as karyotypically normal and the CAG repeat lengths measured using automated genotyping on an ABI 3100 (HD iPSC Consortium, 2012).

\section{Astrocyte Differentiation}

All five lines were first differentiated into neural progenitor cells (NPCs) by growing the cells in suspension in media supplemented with epidermal growth factor and fibroblast growth factor (Ebert et al., 2013). The cultures were mechanically propagated as EZ spheres in growth media [70:30 DMEM:F12 (Life Technologies), 2\% B27 without vitamin A (Life Technologies), 1\% anti-biotic/anti-mycotic (Life Technologies), $100 \mathrm{ng} / \mathrm{ml}$ EGF (Peprotech) and FGF (Peprotech)], as described by Ebert et al. (2009). After a minimum of 10 mechanical passages in weekly intervals, the lines were differentiated to astrocytes using the protocol described below.

Stage 1: At between passages 10 and 30, EZ spheres were dissociated to a single cell suspension using TrypLE (Thermo Fisher Scientific). The ratio of live:dead cells was determined using a hemacytometer by Trypan Blue exclusion. The dissociated NPCs were then plated on poly-L-lysine and laminin-coated glass coverslips at a density of 25,000 live cells per $13 \mathrm{~mm}$ coverslip in 24 -well plates. The cells were seeded in $80 \mu \mathrm{l}$ of base medium [DMEM:F12 1:1, 2\% B27 (Life Technologies) and $1 \%$ antibiotic/antimycotic] and cultured overnight in order increase adherence of the cells to the coverslip. The coverslips were then flooded with the Stage 1 astrocyte differentiation medium [DMEM:F12 1:1, 2\% B27, 1\% antibiotic/antimycotic and $1 \mu \mathrm{M}$ Arabinosylcytosine (Ara-C; Sigma-Aldrich)]. NPCs were cultured for a week in this medium with half medium changes every 3 days.

Stage 2: To remove the Ara- $\mathrm{C}$ after 1 week, the adherent cells were washed twice with base medium before being cultured in the Stage 2 astrocyte medium [DMEM:F12 1:1, 2\% B27, 1\% antibiotic/antimycotic and $10 \mathrm{ng} / \mathrm{ml}$ Lif-1 (Millipore Sigma)] for 1 week, with medium changes every 3 days.

Stage 3: Following 1 week in Stage 2 medium, the cells were dissociated using TrypLE for $5 \mathrm{~min}$ to generate a multi-cell suspension lifted from the coverslip. The cells were then washed in base medium and seeded in T25 Nunc tissue culture flasks. The cells were then fed Stage 3 astrocyte differentiation medium [DMEM:F12 1:1, 8\% heat inactivated fetal bovine serum (hiFBS), $1 \%$ antibiotic/antimyotic and $10 \mathrm{ng} / \mathrm{ml}$ Lif-1], which was changed every 5 days. These cells were considered the "Stage 3" astrocytes 
and were used experimentally. Confluent cells were passaged at a ratio of 1:3, typically every 2 weeks. These Stage 3 cells could be frozen for later experimentation or re-plated onto glass coverslips for immediate study (see below).

Stage 4: The final stage of astrocyte differentiation generated the most functionally mature astrocytes. The Stage 3 cells were dissociated using TrypLE and re-seeded on $13 \mathrm{~mm}$ glass coverslips at a density of 50,000 cells per coverslip (or higher, dependent upon the particular iPSC line). Cells were left overnight in Stage 3 medium before it was changed to Stage 4 medium [DMEM:F12 1:1, 8\% hiFBS, 1\% antibiotic/antimyotic, $10 \mathrm{ng} / \mathrm{ml} \mathrm{Lif-1,} 20 \mathrm{ng} / \mathrm{ml}$ ciliary neurotrophic factor (CNTF; $\mathrm{R} \& \mathrm{D}), 20 \mathrm{ng} / \mathrm{ml}$ bone morphogenic protein -4 (BMP-4; R\&D) and $5 \mu \mathrm{M}$ N-[(3,5-Difluorophenyl)acetyl $]$-L-alanyl-2-phenylglycine-1,1-dimethylethyl ester (DAPT; R\&D)]. If necessary, the cells were cultured longer in Stage 3 medium to achieve confluence prior to the final differentiation in Stage 4 medium, which was important for their survival. After 1 week in this Stage 4 medium, these cells were considered the mature "Stage 4" astrocytes, which were used for the experiments described below.

\section{Neural Differentiation}

iPSC cultures were grown to 70\% confluence on Matrigel-coated plates in mTESR medium (Stemcell Technologies). Using a protocol adapted from a previously published ventral forebrain protocol (Telezhkin et al., 2016), iPSCs were switched to SLI medium [Advanced DMEM/F12 (Invitrogen) 1:1 Neurobasal A (Invitrogen), 1\% Glutamax (Life Technologies), 2\% B27 without vitamin A, $10 \mu \mathrm{M}$ SB-431542 (Tocris), $1.0 \mu \mathrm{M}$ LDN-193189 (Stemgent), $1.5 \mu \mathrm{M}$ IWR1 (Tocris)], and the medium was changed daily. On day 4, $10 \mu \mathrm{M}$ Y-27632 (Abcam) was added to the cultures $1 \mathrm{~h}$ prior to passaging. The cultures were washed with 1X PBS (Corning), treated with accutase (Millipore) for $5 \mathrm{~min}$, agitated to loosen, and centrifuged at $150 \mathrm{x} g$ for $3 \mathrm{~min}$. After the supernatant was aspirated, the cells were resuspended in SLI medium containing $10 \mu \mathrm{M}$ Y-27632 and seeded on a Matrigel-coated plate at a 1:2 density. The cultures were fed daily with SLI medium. On day 8, the cells were passaged 1:2 as described above and seeded onto Matrigel-coated plates in LIA medium [Advanced DMEM/F12 1:1 Neurobasal A, 1\% Glutamax, 2\% B27 without vitamin A, 200 nM LDN-193189, $1.5 \mu \mathrm{M}$ IWR1, $20 \mathrm{ng} / \mathrm{mL}$ Activin A (R\&D)] containing $10 \mu \mathrm{M}$ Y-27632. The cultures were then fed daily with LIA medium. On day 16, the neural progenitor cells (NPCs) were passaged, as described above, and were re-suspended in plating medium (Advanced DMEM/F12 1:1 Neurobasal A, 1\% Glutamax, 2\% B27 without vitamin A) at a concentration of $1 \mathrm{x} ; 10^{6}$ cells $/ \mathrm{mL}$. The neuronal progenitors were then seeded at a density of 25,000 cells per coverslip on either matrigel-coated glass coverslips or glass coverslips previously seeded with astrocytes matured to Stage 4 , as described below. Excess cells ( day 16 of differentiation) were cryopreserved in CryoStor freezing medium for use in later experiments.

\section{Astrocyte Co-culture}

Stage 3 astrocytes were plated and differentiated for 1 week in Stage 4 medium before seeding the NPCs on top. Dissociated
NPCs were plated at a density of 25,000 per coverslip on top of confluent Stage 4 astrocytes. From this point, only neuronal differentiation/maturation media were used, with no further supplements for the astrocytes.

The neuronal/astrocyte co-cultures were maintained using the SynaptoJuice Protocol (Kemp et al., 2016). During the first week, the cells were differentiated in SynaptoJuiceA medium [Advanced DMEM/F12 1:1 Neurobasal A, 1\% Glutamax, 1\% antibiotic/antimyotic, 2\% B27 without vitamin A, $2 \mu \mathrm{M}$ PD0332991 (Tocris), $10 \mu \mathrm{M}$ DAPT, $10 \mathrm{ng} / \mathrm{mL}$ BDNF (Miltenyi), $500 \mathrm{nM}$ LM22A4 (Tocris), $10 \mu \mathrm{M}$ forskolin (Tocris), $3 \mu \mathrm{M}$ CHIR99021 (Tocris), $300 \mu \mathrm{M}$ GABA (Tocris), $1.8 \mathrm{mM}$ $\mathrm{CaCl}_{2}$ (Sigma), $200 \mu \mathrm{M}$ ascorbic acid (Sigma)]. SynpatoJuiceA was changed on day 2 and day 5 after plating. On day 7 post-plating, SynaptoJuice-A was replaced with SynaptoJuice$\mathrm{B}$ differentiation/maturation medium (Advanced DMEM/F12 1:1, Neurobasal A, 2\% Glutamax, 1\% antibiotic/antimyotic, 2\% B27, $2 \mu \mathrm{M}$ PD0332991, $10 \mathrm{ng} / \mathrm{mL}$ BDNF, $500 \mathrm{nM}$ LM22A4, $3 \mu \mathrm{M}$ CHIR99021, $1.8 \mathrm{mM} \mathrm{CaCl}_{2}, 200 \mu \mathrm{M}$ ascorbic acid). SynaptoJuice-B was changed every 3 days for up to day 24 days post-plating, at which time the cultures were fixed for immunocytochemistry.

\section{Immunocytochemistry}

Immunocytochemistry was performed on poly-ornithine coated $13 \mathrm{~mm}$ glass coverslips in 24 well tissue culture plates. Cells were fixed with $4 \%$ paraformaldehyde in phosphate buffered saline (PBS) for $10 \mathrm{~min}$ at room temperature and then rinsed 3 times for 3 min with PBS. Cells were blocked in $10 \%$ bovine serum albumin and $1 \%$ TritonX-100 for $1 \mathrm{~h}$ at room temperature and then incubated overnight at $4^{\circ} \mathrm{C}$ with antibodies diluted in blocking solution. The following antibodies were used: GFAP (rat, Agilent Technologies Cat\# Z0334, RRID:AB_10013382; dilution 1:2500), S100ß (mouse, Sigma-Aldrich Cat\# S2532, RRID:AB_477499; 1:1000), $\beta 3$-tubulin/TuJ1 (rabbit, Sigma-Aldrich Cat\# T2200, RRID:AB_262133; 1:1000), and gamma aminobutyric acid (GABA; rabbit, Sigma-Aldrich Cat\# A2052, RRID:AB_477652; 1:500). Cells were then washed 3 times and incubated for $1 \mathrm{~h}$ at room temperature in green and red fluorescent-conjugated secondary antibodies (Alexa Fluor 448 and Alexa Fluor 594, respectively), diluted at $1: 500$ in blocking solution with $1 \%$ normalized serum from the host species of the secondary antibodies. Cells were then washed 3 times, and incubated with the fluorescent nuclear stain, DAPI, followed by two washes. The coverslip was then mounted on a glass microscope slide using fluoromount-G.

\section{Whole-Cell Patch Clamp}

Whole-cell patch clamp was performed on Stage 3 and Stage 4 astrocyte cultures. Glass micropipettes were pulled using a Sutter Instruments P-1000 from thin wall borosilicate glass capillaries (World precision instruments) with a tip resistance of between 4 and $6 \mathrm{M} \Omega$. Cells were visualized using a Leica DMLED microscope. Voltage and current clamp recordings were performed using a Molecular Devices Multiclamp 700B amplifier, Digidata 1300 converter, and PClamp 10 acquisition software (Molecular Devices). Astrocytes with an access resistance above 
$35 \mathrm{M} \Omega$ were excluded. Cells were bathed in an extracellular solution (ECS) containing (in $\mathrm{mM}$ ): $135 \mathrm{NaCl}, 5 \mathrm{KCl}, 5$ HEPES, 10 Glucose, $1.2 \mathrm{MgCl}_{2}$, and $1.25 \mathrm{CaCl}_{2}$. The internal solution comprised (in mM): $10 \mathrm{NaCl}, 117 \mathrm{KCl}, 11$ HEPES, $2 \mathrm{Na}$.ATP, 2 $\mathrm{MgCl}_{2}$ and $1 \mathrm{CaCl}_{2}$. The cell's capacitance and access resistance was measured using a $10 \mathrm{mV}$ test pulse from a holding voltage of $-70 \mathrm{mV}$. Channel blockers were perfused with a rapid solution changer (BioLogic Science Instruments).

A voltage-step protocol was used both with and without online leak subtraction to record voltage-gated $\mathrm{Na}^{+}\left(\mathrm{Na}_{V}\right)$, voltage-gated $\mathrm{K}^{+}\left(\mathrm{K}_{V}\right)$, inward rectifying $\mathrm{K}^{+}\left(\mathrm{K}_{\text {ir }}\right)$ and tandem pore potassium $\left(\mathrm{K}_{2 P}\right)$ currents. Starting from a holding voltage of $-70 \mathrm{mV}$, a $100 \mathrm{~ms}$ voltage-step was applied, starting at $-120 \mathrm{mV}$ and increasing in $10 \mathrm{mV}$ increments to $+40 \mathrm{mV}$. $\mathrm{Na}_{V}$ currents were observed as rapidly activating and inactivating inward currents immediately following the start of voltage-steps above approximately $-40 \mathrm{mV}$, and were sensitive to $0.5 \mu \mathrm{M}$ Tetrodotoxin (TTX; Tocris). $\mathrm{K}_{V}$ currents were observed as more slowly activating outward currents at voltages more positive than $-20 \mathrm{mV}$ which did not completely inactivate during the $100 \mathrm{~ms}$ step and were sensitive to $10 \mathrm{mM}$ Tetraethylammonium chloride (TEA; Tocris). $\mathrm{K}_{i r}$ currents were observed as a persistent inward current at voltage steps below approximately $-80 \mathrm{mV}$ and were sensitive to $10 \mu \mathrm{M}$ Nortriptyline- and $\mathrm{Ba}^{2+}$-containing ECS [in mM: $95 \mathrm{NaCl}, 5 \mathrm{KCl}, 5$ HEPES, 10 Glucose, $1.2 \mathrm{MgCl}_{2}, 27$ $\mathrm{BaCl}_{2}$ (Sigma-Alrich), 0.01 Nortriptyline hydrochloride (SigmaAldrich)]. $\mathrm{K}_{2 P}$ currents were only observable in the absence of leak subtraction, and were a non-linear, Goldman-Hodgkin-Katz rectifying current which was insensitive to $10 \mu \mathrm{M}$ TEA, but sensitive to $10 \mu \mathrm{M}$ Quinidine (Sigma-Aldrich).

Resting membrane potential (RMP) was assessed in current clamp by averaging from continuous voltage recordings with a $0 \mathrm{pA}$ holding current. Input resistance was measured using $200 \mathrm{~ms}$ current-steps, starting at $+10 \mathrm{pA}$ and increasing in $10 \mathrm{pA}$ increments to $+180 \mathrm{pA}$. The input resistance was then calculated using Ohms law.

\section{Microelectrode Array}

Astrocytes at Stage 3 were thawed into flasks and grown for a week before passage. HD (CS09iHD109n1) and control (CTR; CS83iCTR33n2) astrocytes were then seeded onto 48well transparent microelectrode array (MEA) plates (Axion Biosystems) at between 25 and 200 thousand cells per well. Astrocytes were allowed up to 2 days in Stage 3 media after seeding before switching to Stage 4 media for maturation. After 1 week in maturation, control and HD neural progenitors ( $\sim$ day 16 of differentiation) were seeded on top of the astrocytes at between 50 and 400 thousand cells per well and the co-culture experiment was considered at Day 0 . Daily recordings of activity were measured using the Maestro MEA platform and recording software (Axion Biosystems) for $5 \mathrm{~min}$ durations. Wave form events were identified using adaptive spike threshold crossing with a standard deviation of noise set at 5.5, and then further validated and sorted to individual neurons using Offline Sorter (Plexon). A minimum of 1 spike per min was used to include neuronal waveforms for analysis, and PCA analysis of each unit cluster was used to exclude noise artifacts. Data was compiled in excel and statistics run using GraphPad Prism 7.04 and SigmaPlot software.

\section{ELISA}

A standard dilution curve (500 to $7.8 \mu \mathrm{g} / \mathrm{mL}$ ) was generated by serial dilution of human thrombospondin-1, with a negative control of assay diluent. Each astrocyte-conditioned medium sample was assayed at full and half concentration, diluted in base medium, and additionally the base medium was sampled as a negative control. Thrombospondin-1 was quantified in astrocyte-conditioned medium using a human thrombospondin-1 Quantikine ELISA Kit (R\&D Systems), following the manufacturer's instructions. The plate was read at an excitation wavelength of $450 \mathrm{~nm}$, simultaneously correcting for a $570 \mathrm{~nm}$ background signal. The generated standard curve was then used to calculate the concentration of thrombospondin-1 in astrocyte conditioned medium.

\section{Cell Counts}

Fluorescent imaging of astrocyte cultures was performed on a Leica microscope, model DM6000 B. Images were taken from DAPI (400 nm), GFP (470 nm), Texas red (560 nm), and Y5 $(620 \mathrm{~nm})$ channels using 10x; and 20x; air objectives. Images were acquired using Leica Application Suite X (Leica Microsystems). Several random and representative images were taken per coverslip and manually counted using ImageJ. Cell bodies, or appropriate cell regions staining positive for cell markers, were counted and expressed as a ratio of the total DAPI-positive cell nuclei counts.

Cells in the neuronal cultures were noted to have much smaller nuclei compared to those in the astrocyte cultures. Nuclei were measured after 16 days of glial differentiation followed by 6 days in neuronal media with or without co-culture. Nuclei size was measured with the Zeiss stereological software nucleator feature using 8 rays, 1 marker per interval. Neuronal cultures and neuronal-astrocyte co-cultures were quantified (at 6 days post-plating) with Zeiss stereological investigator software using a 20x air objective. Cells in the co-cultures were divided into two groups based upon their nuclei size as a means of distinguishing whether they originated from the neuronal or astrocyte differentiation protocols.

\section{$\mathrm{Ca}^{2+}$ Imaging}

Six days post-co-culture plating and maintenance in neuronal media, HD (CS09iHD109n1) and control (CS83iCTR33n2) cells were incubated with $5 \mu \mathrm{M}$ Fluo-3-AM (Fluo-3) for $30 \mathrm{~min}$ in their culture medium at $37^{\circ} \mathrm{C}$. Excess Fluo- 3 was then washed out and replaced with fresh media. Fluo-3 was excited at $490 \mathrm{~nm}$ and emission was recorded at $530 \mathrm{~nm}$ using a Leica microscope model DM6000 B equipped with a DFC360FX CCD camera. Excitation exposure was between 25 to $35 \mathrm{~ms}$ with a gain between 5 and $8 x$, depending upon region of interest with an acquisition rate of $15.5 \mathrm{~Hz}$. Fluorescence intensity was measured over time in several regions of interest on both astrocytes and neurons using ImageJ software. Off-line subtraction was performed by measuring fluorescence in a region of interest which did not contain cells or cell extensions. Astrocyte- or 
neuron-dependent events were discriminated by correlating the cell morphology (using transmitted light) with duration of $\mathrm{Ca}^{2+}$ response, and further confirmed by the addition of TTX to block $\mathrm{Na}_{v}$-dependent action potentials in neurons.

\section{Glutamate Response and LDH Activity}

For each of three experiments, four assays were performed, beginning on day 1 after transition to SynaptoJuice B, and each assay was separated by $4-5$ days over the 16-day culture duration. Spontaneous MEA recordings were taken up to $1 \mathrm{~h}$ before addition of glutamate. Glutamate $(100 \mu \mathrm{M})$ was then added to each well of the 48-well plate and the neural activity was immediately recorded (note: Advanced DMEM/F12 contains $0.05 \mathrm{mM}$ glutamate). The plate was then incubated for $12 \mathrm{~h}$ at $37^{\circ} \mathrm{C}$, followed by a recording of activity of the neurons under chronic glutamate exposure. Media was sampled from every well and pooled by cell group (control astrocytes alone, HD astrocytes alone, control astrocytes with control neurons or HD neurons, and HD astrocytes with control neurons or HD neurons). The concentration of lactate dehydrogenase (LDH) was then measured using a Pierce LDH cytotoxicity assay kit (Thermo Fisher Scientific) per the manufacturer's protocol.

\section{Statistics}

Chi Squared tests were performed on data with a categorical or binomial dependent variable. $T$-tests were performed on data with a continuous dependent variable, following inspection to be sure that the data were normally distributed around each hypothesized mean. Depending on the distribution of the data set, either a parametric or non-parametric analysis of variance was performed on data sets with more than two groups. Nuclei sizes were compared with a one-way ANOVA followed by a Bonferroni post-test. Outliers were excluded from MEA data of spontaneous activity if they exceeded \pm 2 standard deviations from the mean of three experiments. Post hoc tests were then chosen according to the data sets and are stated. Unless stated otherwise, continuous data are reported as mean \pm standard error of the mean.

\section{RESULTS}

\section{Human iPSCs Provide a Pure Population of Astrocytes That Produce Hallmark Proteins}

To examine the influence of $\mathrm{HD}$ on astrocytes, astrocytes were differentiated from previously described control (18, 21, or 33 CAG repeats) and HD patient (77 or 109 CAG repeats) iPSC lines (HD iPSC Consortium, 2012). The differentiation protocol consisted of four separate, week-long "stages" that were specifically chosen to rapidly generate mature astrocytes (Figure 1A). In Stage 1, iPSCderived NPCs were treated with $1 \mu \mathrm{M}$ Ara-C, which inhibits cell division. This was used as neural progenitors require cell division for neurogenesis (Nelson et al., 2008), thereby low doses of Ara-C were used to block neuronal generation. Compared to untreated NPCs, cultures treated with Ara-C contained little to no TuJ-positive neurons (Supplementary Figure 1). In Stage 2, Ara-C was replaced by Lif-1 to induce astrogliogenesis (Wright et al., 2003). In Stage 3, cells were switched into a Lif- 1 and high fetal bovine serum (FBS)-containing medium to elicit astrocyte proliferation. Finally, in Stage 4, cells were switched into medium containing further astrogliogenic factors (BMP-4 and CNTF), as well as a $\gamma$-secretase inhibitor (DAPT) to encourage synchronous terminal differentiation and maturation (Telezhkin et al., 2016).

Immunocytochemistry assessment of Stages 3 and 4 astrocytes, from both control and HD iPSC lines, showed a typical large filamentous astrocyte morphology as well as expression of the known astrocyte markers glial fibrillary acidic protein (GFAP) and S100 $\beta$ (Figure 1B). The relative expression of these two proteins can indicate the degree of astrocyte maturation, with GFAP expression present in neural precursors and $S 100 \beta$ expression present in more mature astrocytes (Raponi et al., 2007). Quantifying the percent of total cells expressing astrocyte markers showed that Stages 3 and 4 cultures had a high percentage of both $\mathrm{GFAP}^{+}$and/or S100 $\beta^{+}$cells (Figure 1C). During the transition from Stage 3 to Stage 4, both control and HD lines had a significant loss of GFAP-positive/ S100 $\beta$-negative cells ( $p<0.001$; Figure 1C). HD and control cells both expressed markers of mature astrocytes in similar proportions at Stages 3 and 4.

The secretion of neurogenic and synaptogenic factors by astrocytes is critical to their function, and pertinent for co-culture studies using astrocytes to enhance neuronal differentiation. Thombospondin-1 (TSP1) is one such synaptogenic factor generated by astrocytes in the developing central nervous system (Chung et al., 2015). To determine the relative functionality of iPSC-derived astrocytes, a TSP1 ELISA was performed on medium conditioned by astrocytes for $24 \mathrm{~h}$. Results revealed a significant increase in TSP1 secretion from Stage 3 to Stage $4(p<0.001$; Figure 1D), which was not due to cell number differences as subsequent cell fixation and counts confirmed similar cell numbers between Stages 3 and 4 cultures (data not shown). The increased TSP1 secretion from Stage 4 astrocytes supports that the final differentiation step generates functionally mature astrocytes. Similar GFAP and $S 100 \beta$ protein production, HD and control astrocytes in Stages 3 and 4 have no difference in TSP1 levels.

\section{Human iPSCs Provide a Range of Functionally Mature Astrocytes}

In addition to protein expression (Raponi et al., 2007), glial fate can be validated by specific electrophysiological characteristics. Functional traits are cell-specific and demonstrate quantifiable ontogenies during development through to maturity (Shelton and McCarthy, 1999; Zhou et al., 2006). Specifically, immature astrocytes display significant $\mathrm{K}_{v}$ currents and even small $\mathrm{Na}_{v}$ currents, reminiscent of very immature neurons (Zhou et al., 2006). As astrocytes mature, they express progressively larger $K_{i r}$ and $\mathrm{K}_{2 P}$ currents (Higashi et al., 2001; Zhou et al., 2006).

To establish the functional maturity of the Stages 3 and 4 astrocytes, we employed whole-cell patch clamp 
A
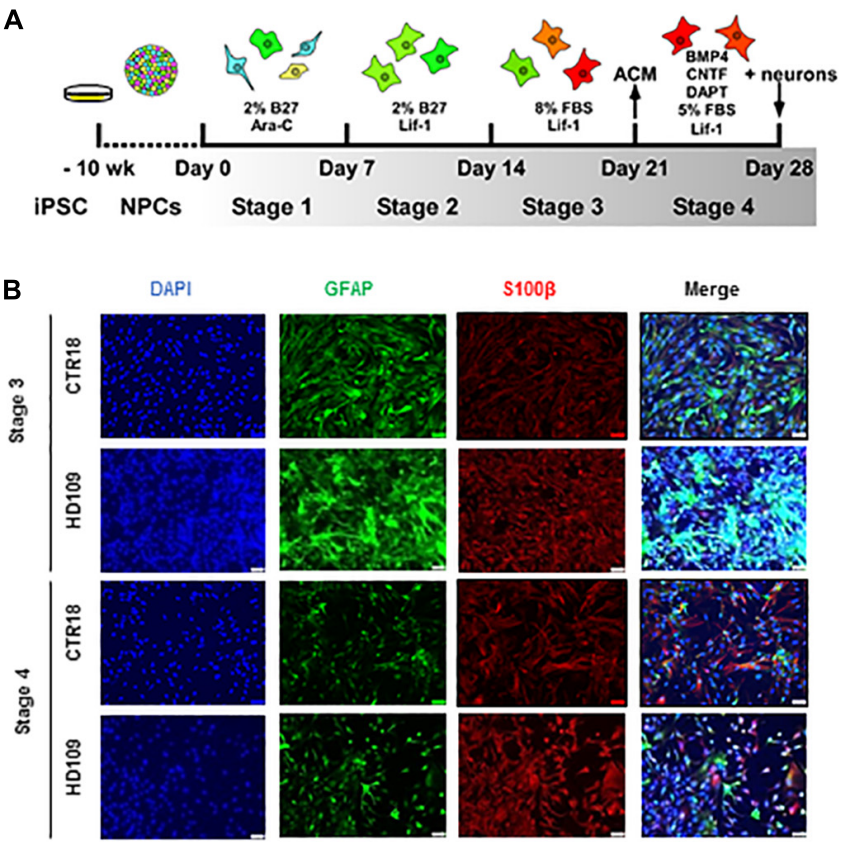
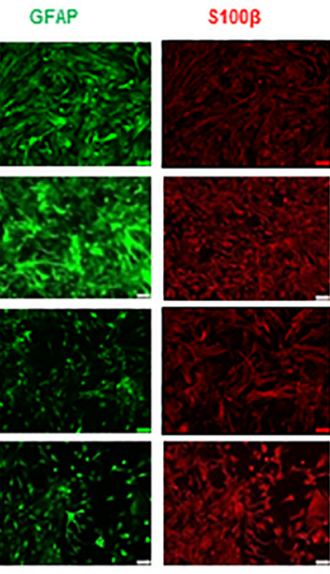

C

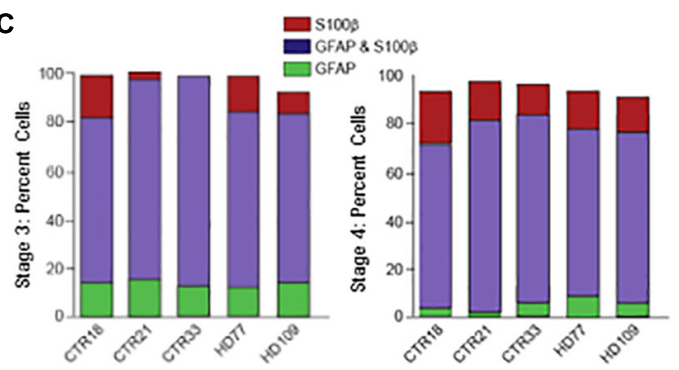

D

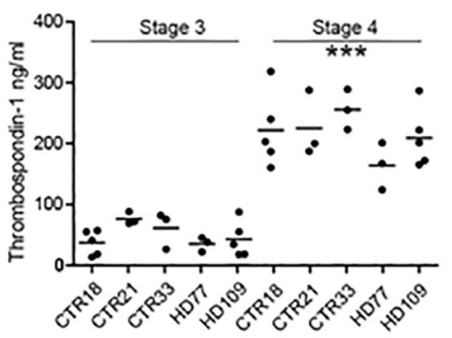

FIGURE 1 | Huntington's disease and control iPSCs are equally capable of generating mature astrocytes. (A) Schematic of astrocyte differentiation from iPSC-derived neurospheres. (B) Immunocytochemistry of Stages 3 and 4 astrocytes stained for GFAP and S100ß. (C) Stage 3 (left) and Stage 4 (right) display astrocyte markers GFAP and/or S100 $\beta$ in equal percentages between HD and controls. ( $n=$ at least three independent experiments per line and more than 1,900 DAPI-positive cells). (D) Stage 4 astrocytes derived from either HD or control iPSCs release more TSP1 than Stage 3 astrocytes in all lines tested, indicative of increased maturity (*** $p<0.001 ; n=3-5$ independent differentiations).

electrophysiology to measure ionic currents that are fundamental to astrocyte function. Four electrophysiological types of astrocytes were identified in our cultures and were termed Types A through D. Type A (Figure 2A, left) astrocytes were characterized as having both $\mathrm{K}_{v}$ and $\mathrm{Na}_{v}$ currents. Highly immature astrocytes and astrocyte precursors generate very small voltage-gated $\mathrm{K}^{+}$and, if present, voltage-gated $\mathrm{Na}^{+}$currents (Zhong et al., 2016). While this suggests immature neurons, we have shown that these cells are almost entirely $\mathrm{GFAP}^{+}$ and/or S100 $\beta^{+}$(Figure 1B) and have little to no observed TuJ1 staining (Supplementary Figure 1). Collectively, these phenotypes suggest that Type A cells are precursor astrocytes rather than neurogenic. Type $\mathrm{B}$ astrocytes expressed only $\mathrm{K}_{v}$ currents (Figure 2A, right), also indicative of an immature astrocyte progenitor. Pharmacological analysis of the ion channels in Type A and B astrocytes demonstrated the presence of Tetraethylammonium chloride (TEA)-sensitive Kv currents (Figure 2B), as would be expected for a progenitor (Lai et al., 2010). When examining the $\mathrm{K}^{+}$current densities between the immature (Type A and $\mathrm{B}$ ) control and $\mathrm{HD}$ astrocytes, no differences were observed (CTR: $20.93 \pm 0.92 \mathrm{pA} / \mathrm{pF}, \mathrm{HD}$ : $22.12 \pm 0.98 \mathrm{pA} / \mathrm{pF}, n=23 \mathrm{CTR}, 24 \mathrm{HD}$; Figure 2C). To further investigate any $\mathrm{HD}$-related differences in this population of immature astrocytes, we next examined their passive membrane properties. The resting membrane potential (RMP) in glia is important for the control of cell excitability, and contributes to the buffering of extracellular $\mathrm{K}^{+}$and uptake of neurotransmitters (McKhann et al., 1997). The RMP was lower in HD compared to control (CTR: $-51.24 \pm 1.0 \mathrm{mV}, \mathrm{HD}:-57.30 \pm 0.90 \mathrm{mV}, n=56$ CTR, $24 \mathrm{HD} ; p<0.0001$; Figure 2D). Input resistance, a crude measure of the concentration of ion channels present on the cell surface, was higher in the HD astrocytes (CTR: $1036 \pm 26.18$ $\mathrm{M} \Omega, \mathrm{HD}: 1188 \pm 27.49 \mathrm{M} \Omega, n=56 \mathrm{CTR}, 30 \mathrm{HD} ; p=0.0001$; Figure 2E). And cell capacitance, a precise measure of the cell surface area, was smaller in HD lines compared to controls (CTR: $27.57 \pm 0.71 \mathrm{pF}, \mathrm{HD}: 23.40 \pm 0.90 \mathrm{pF}, n=54 \mathrm{CTR}, 30 \mathrm{HD}$; $p=0.0007$; Figure 2F).

Over the course of astrocyte development and maturation, $\mathrm{K}_{i r}$ channels are expressed (Seifert et al., 2009), with $\mathrm{K}_{i r^{-}}$ positive astrocytes present at higher concentrations in the developing brain. Corroborating this in vivo phenotype, another electrophysiological phenotype present in the Stage 3/4 cultures, termed Type $\mathrm{C}$ astrocytes, expressed $\mathrm{K}_{i r}$ currents (Figure 3A). Furthermore, pharmacological analysis showed that Type $\mathrm{C}$ astrocytes expressed TEA-resistant, nortriptyline-sensitive $\mathrm{K}_{i r} 4.1$ channels (Figure 3B). While Type A/B astrocytes had similar $\mathrm{K}_{V}$ current densities between $\mathrm{HD}$ and control lines, Type $\mathrm{C}$ $\mathrm{HD}$ astrocytes expressed smaller peak $\mathrm{K}_{\text {ir }}$ currents compared to controls (CTR: $4.25 \pm 0.22 \mathrm{pA} / \mathrm{pF}, \mathrm{HD}: 3.01 \pm 0.21 \mathrm{pA} / \mathrm{pF}, n=13$ CTR, $15 \mathrm{HD} ; p<0.0001$; Figure 3C). HD Type $\mathrm{C}$ astrocytes also differed in passive membrane properties, including depolarized RMP values (CTR: $-74.93 \pm 0.70 \mathrm{mV}$, HD: $-68.82 \pm 0.93 \mathrm{mV}$, $n=14 \mathrm{CTR}, 14 \mathrm{HD} ; p<0.0001$; Figure 3D), greater input resistances for $\mathrm{HD}$ astrocytes (CTR: $570.20 \pm 36.27 \mathrm{M} \Omega$, HD: $765.7 \pm 25.2 \mathrm{M} \Omega, n=17 \mathrm{CTR}, 14 \mathrm{HD} ; p=0.0001$; Figure 3E) and less capacitance (CTR: $34.59 \pm 1.34$ pF, HD: $28.36 \pm 1.14$ 

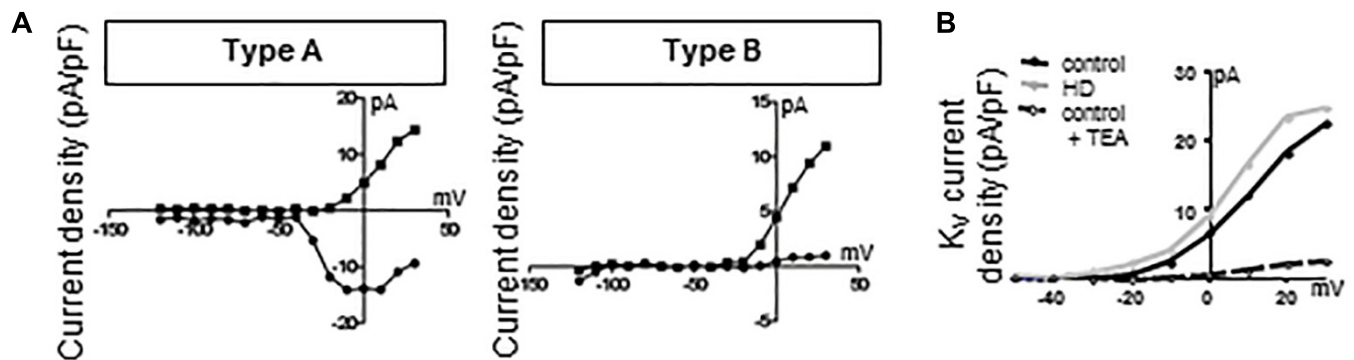

C

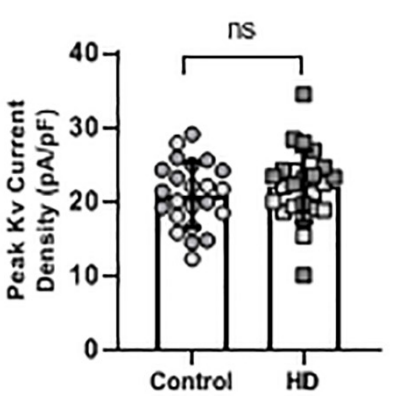

D

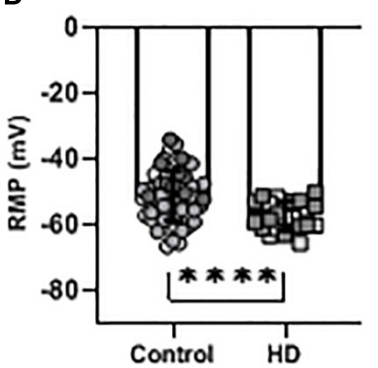

E

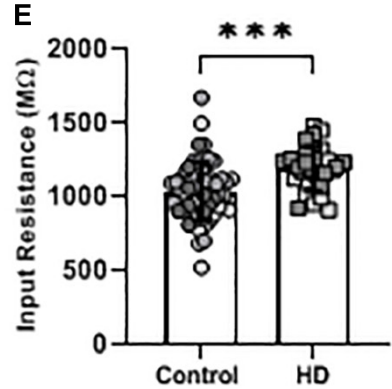

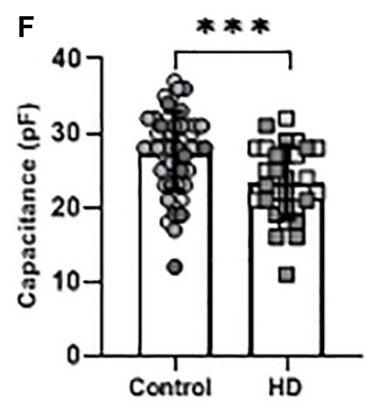

OCTR18 OCTR21 OCTR33 $\square$ HD77 $\square$ HD109

FIGURE 2 | Stages 3 and 4 HD iPSC-derived astrocytes display reduced capacitance as committed but immature Type A/B astrocytes. (A) iPSC-derived astrocytes mature from astrocyte progenitors expressing voltage-gated $\mathrm{Na}^{+}$and $\mathrm{K}^{+}$currents (Type $\mathrm{A}$, left), to immature astrocytes expressing some voltage-gated $\mathrm{Na}^{+}$but mostly voltage-gated $\mathrm{K}^{+}$currents (Type B, right). (B) Example current-voltage (IV) curve of voltage-gated $\mathrm{K}^{+}$current density from HD, control and control + TEA in Type A/B astrocytes. (C) Immature astrocytes display no differences in the peak voltage-gated $\mathrm{K}^{+}$current density between HD and control. (D) Type A/B HD astrocytes have more hyperpolarized resting membrane potentials compared to control and (E) higher input resistance. (F) Immature HD astrocytes show smaller capacitances compared to controls. Each cell is represented as a single point, and therefore $\mathrm{N}$ is represented on each graph. These cells were analyzed from more than three separate experiments $\left(^{* * *} p<0.005\right.$ and $\left.{ }^{* * * *} p<0.001\right)$.

$\mathrm{pF}, n=17 \mathrm{CTR}, 14 \mathrm{HD} ; p=0.0014$; Figure 3F) compared to control lines.

Astrocytes within the adult brain predominantly display large $\mathrm{K}_{2 P}$ currents (Seifert et al., 2009), which were identified in the final electrophysiological phenotype present in the Stage 3/4 cultures, termed Type D astrocytes (Figure 3G). iPSCderived Type $\mathrm{D}$ astrocytes expressed quinidine-sensitive $\mathrm{K}_{2 P}$ channels that indicate a mature astrocytic fate (Figure $3 \mathbf{H}$ ). These astrocytes did not demonstrate any difference in the peak $\mathrm{K}^{+}$current density between $\mathrm{HD}$ and control lines (CTR: $7.06 \pm 0.38 \mathrm{pA} / \mathrm{pF}, \mathrm{HD}: 7.01 \pm 0.35 \mathrm{pA} / \mathrm{pF}, n=12 \mathrm{CTR}, 13$ HD; Figure 3I). Similarly, passive membrane properties such as RMP (CTR: $-77.07 \pm 0.73 \mathrm{mV}$, HD: $-76.29 \pm 0.83 \mathrm{mV}$, $n=15 \mathrm{CTR}, 13 \mathrm{HD}$; Figure 3J) and input resistance (CTR: $372.50 \pm 20.78 \mathrm{M} \Omega, \mathrm{HD}: 361.20 \pm 23.50 \mathrm{M} \Omega$, $n=12 \mathrm{CTR}, 13 \mathrm{HD}$; Figure $3 \mathrm{~K}$ ) were not different between HD and control lines. However, HD Type D astrocytes had lower capacitance values compared to controls (CTR: $41.13 \pm 1.44 \mathrm{pF}, \mathrm{HD}: 32.81 \pm 1.25 \mathrm{pF}, n=15 \mathrm{CTR}, 13 \mathrm{HD}$; $p=0.0002$; Figure 3L).

Collectively, these results demonstrate that this new protocol can differentiate both control and HD iPSC lines into a population of primarily pure astrocytes, and that these astrocytes vary in maturity levels that can be characterized by different electrophysiological properties. Astrocytes “Type A-D” are indicative of increasing maturity levels and, indeed, a detailed analysis of passive properties showed differences between the astrocyte Types driven by maturation (Supplementary Figure 2). While in stage 3 media, only Type A $(34.4 \pm 8.9 \%$ total cells) and Type B (65.6 $\pm 8.9 \%)$ astrocytes were observed (Supplementary Figure 2A). However, when cultured in the final, stage 4 media which promotes functional maturation of the astrocytes, a significant drop in Type A astrocytes was observed (3.2 $\pm 3.1 \%$ total cells), while the proportion of mature Type $\mathrm{C}$ and $\mathrm{D}$ astrocytes increased (Type B: $39.4 \pm 2.73 \%$, Type C: $31.2 \pm 3.57 \%$; Type D: $26.0 \pm 1.92 \%$ total cells; Supplementary Figure 2A). Type $\mathrm{C}$ and $\mathrm{D}$ astrocytes demonstrated hyperpolarized RMP (Type A\&B: $-53.10 \pm 2.25 \mathrm{mV}$, Type C: $-72.41 \pm 1.53 \mathrm{mV}$, Type D: $-77.10 \pm 0.61 \mathrm{mV}, n=1$ mean value per line, 5 lines; $p<0.0001$; Supplementary Figure 2B), decreased input resistance (Type A\&B: $1090 \pm 49.76 \mathrm{M} \Omega$, Type C: $651.40 \pm 48.94 \mathrm{M} \Omega$, Type D: $369.50 \pm 9.51 \mathrm{M} \Omega$; $p<0.0001$; Supplementary Figure 2C) and larger cell capacitance (Type A\&B: $25.65 \pm 1.219$ pF, Type C: $32.12 \pm 1.674$ pF, Type D: $38.99 \pm 3.08$ pF; Type D vs. Type A\&B $p=0.0028$; Supplementary Figure 2D) when compared to the immature Type A/B astrocytes. Additionally, the most mature Type D astrocyte had a lower input resistance compared to Type $\mathrm{C}$ astrocytes $(p=0.0011)$. This maturation in 


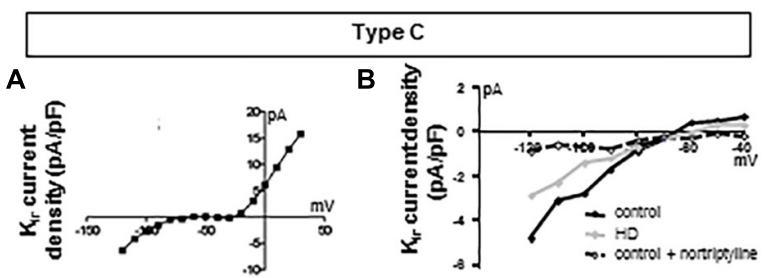

C
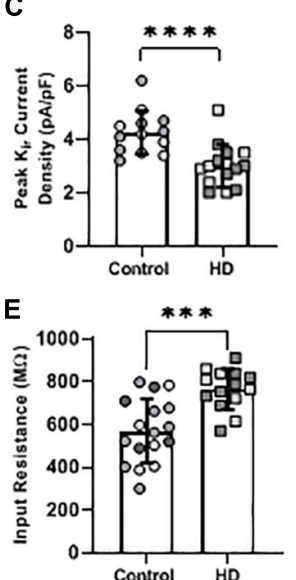

D

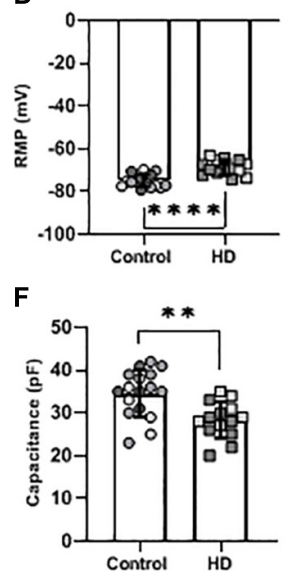

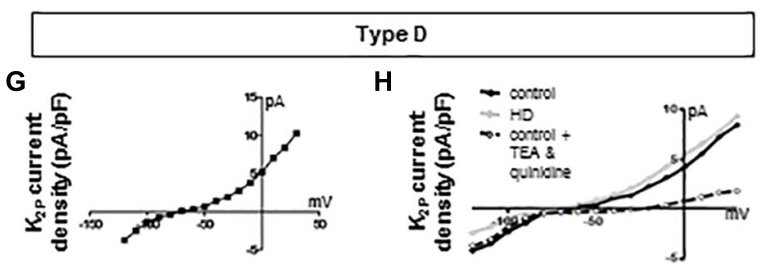

I

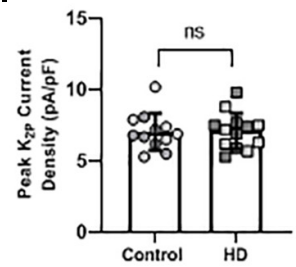

J

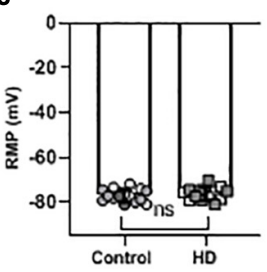

K

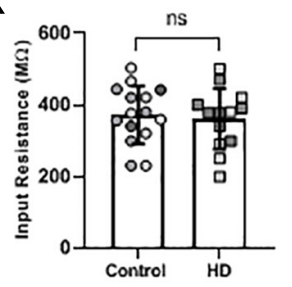

$\mathbf{L}$

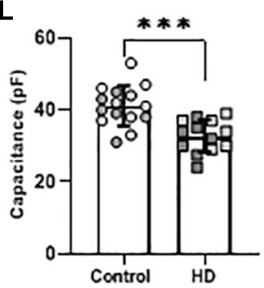

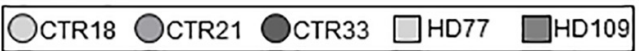

FIGURE 3 | Huntington's disease iPSC-derived $\mathrm{K}_{\text {ir }}$ channel-expressing (Type C) astrocytes display different current densities, resting membrane potential, input resistance and capacitance. (A) Type $\mathrm{C}$ astrocytes express $\mathrm{K}_{i r}$ currents in Stages 3 and 4 cultures. (B) Example IV curve from Type C HD, control and control + nortriptyline astrocytes. (C) Type C HD astrocytes display significantly less peak $\mathrm{K}_{\mathrm{ir}}$ current density as compared to controls. (D) Type C HD astrocytes have depolarized resting membrane potentials and (E) an increased input resistance compared to controls. (F) Type C HD astrocytes have decreased capacitance compared to controls. (G) Mature Type D astrocytes express $\mathrm{K}_{2 P}$ currents in Stages 3 and 4 cultures. (H) Example IV curve of $\mathrm{K}^{+}$current density from Type D HD, control and control + TEA and quinidine astrocytes. (I) Peak $K_{2 P}$ current density, (J) resting membrane potential, and (K) input resistance are similar between HD and control Type D astrocytes. (L) Type $\mathrm{D}$ astrocytes have smaller capacitances than control. Each cell is represented as a single point, and therefore $\mathrm{N}$ is represented on each graph. These cells all came from more than three separate experiments $\left({ }^{* *} p<0.01 ; * * * p<0.005 ;\right.$ and $\left.{ }^{* * * *} p<0.0001\right)$.

electrophysiological properties was conserved across HD and control astrocytes.

\section{HD Astrocytes Are Less Supportive of Neuronal Spontaneous Electrophysiological Activity Than Control Astrocytes as Evidenced by $\mathrm{Ca}^{2+}$ Imaging Experiments}

Because the HD109 line demonstrated more pathotypes than the HD77 line, this more severe HD patient line was used in subsequent experiments and compared to the CTR33 line. As astrocytes promote neuronal development (Pfrieger and Barres, 1997; Pfrieger, 2010), the contribution of HD astrocytes to neuronal physiology was assessed by co-culturing Stage 4 HD astrocytes with iPSC-derived striatal neurons (Telezhkin et al., 2016). To provide functional references, HD and control astrocytes and neurons were also analyzed alone in monocultures. Spontaneous intracellular $\mathrm{Ca}^{2+}$ waves are indirect measures of activity in astrocytes and synaptic firing in neurons, and so $\mathrm{Ca}^{2+}$ imaging was conducted after 6 days of coculture. Neuronal events, observed in the small round cell bodies (Supplementary Figure 3A), were of short duration but involved a large change in fluorescent intensity from baseline levels (Figure 4A). Astrocytic transients, observed in the large cell bodies (Supplementary Figure 3B), were of significantly longer duration and had a smaller change in emission intensity (Figure 4B). To confirm the identity of these spontaneous events, TTX was used at the end of recordings to block the $\mathrm{Na}_{v^{-}}$ dependent neuronal action potentials (data not shown).

Co-culture with control or HD astrocytes led to a striking increase in the percent of neurons with spontaneous events compared to neurons cultured alone ( $p<0.01$; Figure 4C). In addition, the frequency of events increased when neurons were co-cultured with control and HD astrocytes $(p<0.001$; Figure 4D). Of note, however, the co-cultured HD astrocytes were significantly less supportive of spontaneous events and the frequency of events in control neurons ( $p<0.05$; Figures 4C,D) compared to control astrocytes. This suggests that while both astrocytes are supportive of neurons, the HD astrocytes are less supportive of control neurons compared to control astrocytes.

When we examined $\mathrm{Ca}^{2+}$ waves in the iPSC-derived astrocytes, we saw an increase in the proportion of astrocytes exhibiting spontaneous transients when co-cultured with 


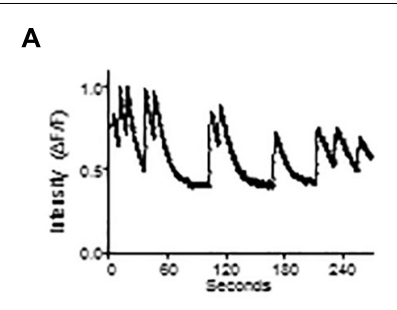

B

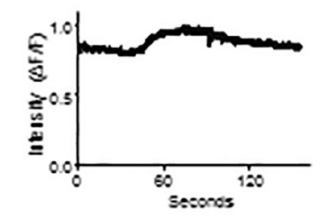

H

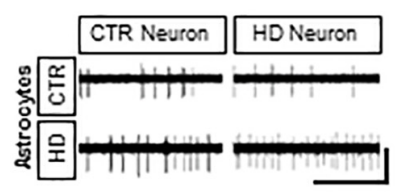

C

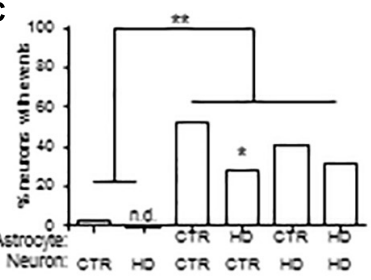

E

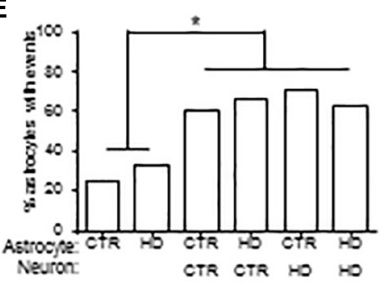

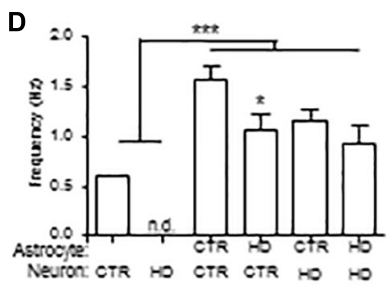

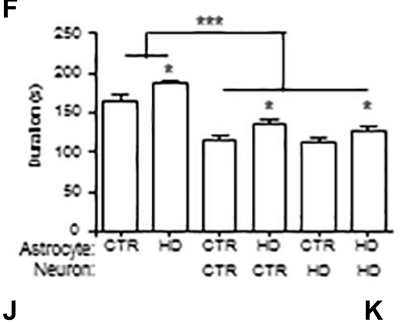

$\checkmark$
G

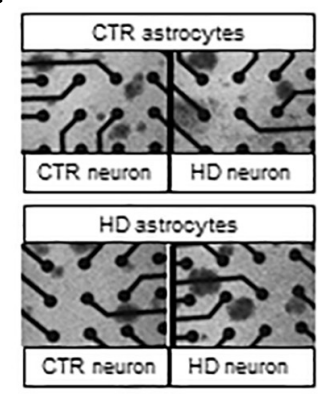

I
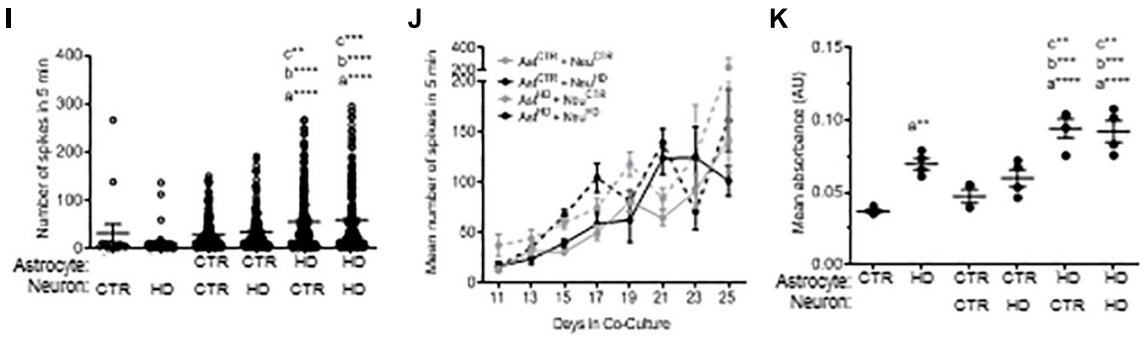

FIGURE 4 | Co-cultures of HD (HD109) and control (CTR33) astrocytes and neurons demonstrate alteration in neuronal firing at early time points and increased toxicity in response to glutamate. (A) Example traces of calcium events in control neurons with control astrocytes. (B) Example traces of calcium events in control astrocytes with control neurons. (C) Percentage of neurons with calcium events is higher in co-culture, but lower in HD astrocyte co-culture compared to control astrocyte co-culture. (D) Frequency of events is higher in neurons in co-culture compared to mono-culture, but less frequent in HD astrocyte co-culture compared to control astrocyte co-culture. (E) Percentage of astrocytes with calcium transients is greater in co-culture compared to mono-culture. (F) Astrocytes in mono-culture had longer transients than in co-culture, and HD astrocytes in mono-culture had longer transients than control astrocytes. Co-culture of control or HD neurons with HD astrocytes showed a longer duration of calcium transients compared to co-culture with control astrocytes. (G) Representative images of astrocyte and neuron co-cultures around the 16 electrodes (black circles) of a 48-well MEA plate. (H) Example electrode traces showing spikes detected from control (CTR) or HD neurons that are co-cultured with CTR or HD astrocytes. Scale bar: $12.5 \mu \mathrm{V}$ vertical by $5 \mathrm{~s}$ horizontal. (I) Spontaneous activity from control and HD co-cultures. Significance between conditions denoted as "a": from Neu ${ }^{H D}$, "b": from Neu ${ }^{\text {CTR }}+\mathrm{Ast}^{\mathrm{CTR}}$, "c": from Ast ${ }^{\mathrm{CTR}}+\mathrm{Neu}^{\mathrm{HD}}$. (J) No difference in total mean spike number per neuron in co-culture conditions during daily 5 min recordings over the course of 16 days. Data is presented as separated by the neuron co-culture (gray lines, control neurons; black lines, HD neurons), with solid lines representing control astrocytes and dashed lines indicating HD astrocytes. (K) Mean absorbance values of LDH activity in media after $12 \mathrm{~h}$ incubation with $100 \mu \mathrm{M}$ glutamate at 4 time points over 16 days. Significance between conditions denoted as "a": from Ast CTR, "b": from Ast ${ }^{\text {CTR }}+$ Neur CTR, "c": from Ast ${ }^{\text {CTR }}+\mathrm{Neu}^{\mathrm{HD}}$. Ast, astrocyte; Neu, neuron; CTR, control. Calcium imaging: cells were analyzed from more than three separate experiments using a Chi squared test. MEA: activity was analyzed using Kruskal-Wallis ANOVA on ranks with Dunn's method from three experiments with 8-66 neurons per condition at each time point. LDH, Analysis on data from triplicates measured at each of four time points across three experiments using one-way ANOVA with Bonferroni correction for multiple comparison $\left({ }^{*} p<0.05,{ }^{* *} p<0.01,{ }^{* * *} p<0.005\right.$, and $\left.{ }^{* * * *} p<0.0001\right)$.

neurons $(p<0.05$; Figure 4E). Although no difference was observed in the amount of transient activity in monoculture, the duration of transients in HD astrocytes was longer compared to control astrocytes $(p<0.05$; Figure 4F). Further, co-culture with neurons decreased the duration of transients in both HD and control astrocytes ( $p<0.001$; Figure $4 F$ ), which suggests the neuron co-culture enhances the speed of intracellular calcium signaling within astrocytes. However, in co-culture with either control or HD neurons, the HD astrocytes generated longer events compared to those in control astrocyte co-cultures $(p<0.05$; Figure 4F). This is a novel phenotype observed in $\mathrm{HD}$ astrocytes, which could be the result of impaired $\mathrm{Ca}^{2+}$ modulation in HD iPSC-derived astrocytes, similar to what has been reported in HD neurons (Lim et al., 2008).

To expand upon the $\mathrm{Ca}^{2+}$ imaging results and evaluate the effects of co-culture over time, we employed microelectrode array (MEA) analysis of Stage $4 \mathrm{HD}$ and control astrocytes cocultured with HD and control neurons (Figure 4G). MEA allows for non-invasive recordings of extracellular action potentials generated by neuronal activity (Spira and Hai, 2013), which we quantified as the mean spike number per neuron over $5 \mathrm{~min}$. First, we compared mono-culture and co-culture of $\mathrm{HD}$ and control astrocytes and neurons from days 9-13 (Figure 4H). As seen via calcium imaging, no difference was observed in the activity between HD and control neurons in mono-culture, while co-culture with astrocytes caused an increase in activity. In contrast with calcium imaging, we did not observe lower activity levels in neurons co-cultured with HD astrocytes. Instead, co-culture of HD astrocytes with either control or HD neurons caused an increase in spiking activity compared to that observed with control astrocytes (Neuro ${ }^{\text {CTR }}$ : $48.49 \pm 14.53$, Neuro $^{\mathrm{HD}}: 47.47 \pm 24.50$, Neuro ${ }^{\mathrm{CTR}}+$ Ast $^{\text {CTR }}: 63.12 \pm 14.64$, 
Neuro $^{\mathrm{HD}}+\mathrm{Ast}^{\mathrm{CTR}}: \quad 68.89 \pm 15.21, \quad \mathrm{Neuro}^{\mathrm{CTR}}+\mathrm{Ast}^{\mathrm{HD}}$ : $96.43 \pm 21.80$, Neuro ${ }^{\mathrm{HD}}+\mathrm{Ast}^{\mathrm{HD}}: 84.45 \pm 17.37$ spikes $/ 5 \mathrm{~min}$, $n=15$ to 207 neurons per condition, three separate experiments; $p<0.001, p<0.005$, and $p<0.0001$; Figure 4H). The contrast between calcium imaging data and MEA activity is likely due to the later time points of analysis in MEA (an additional 5 days in co-culture), which could allow for neurons to mature further. However, when the activity was observed over the entire 25-day duration, no differences between the HD and control astrocyte co-cultures could be seen ( $n=8$ to 166 neurons per time point, three separate experiments; $p=0.293$; Figure 4I). This demonstrates that the initial decreased activity in control neurons that are co-cultured with HD astrocytes observed with calcium imaging, and then increase in activity of both control and $\mathrm{HD}$ neurons that are co-cultured with $\mathrm{HD}$ astrocytes, are transient phenotypes that attenuate over time in co-culture, as evident by the longer duration MEA recordings.

\section{HD Astrocytes Cannot Protect Against Glutamate-Mediated Neuronal Toxicity}

Decreased glutamate re-uptake is a component of the dysfunction with striatal HD astrocytes (Bradford et al., 2009; Faideau et al., 2010; Khakh et al., 2017). As such, we next assessed glutamateinduced toxicity in astrocytes and co-cultures by measuring lactate dehydrogenase (LDH) activity in the culture medium after a 12-h incubation with excess $(150 \mu \mathrm{M})$ glutamate (Figure 4K). When cultured alone, the HD astrocytes had higher levels of LDH activity compared to control astrocytes (Ast ${ }^{\text {CTR }}$ : $0.037 \pm 0.001$, Ast $^{\mathrm{HD}}: 0.070 \pm 0.004$ absorbance, $n=4$ assays per each of three experiments; $p<0.001)$. Indeed, co-culture of control astrocytes with either HD or control neurons did not increase the levels of LDH activity over that of control astrocytes alone. This suggests that with control astrocyte co-culture, neurons of either genotype do not cause an increase in toxicity in response to glutamate. However, in the presence of $\mathrm{HD}$ astrocytes, toxicity levels were increased with co-culture of both control and HD neurons compared to control astrocyte cocultures, respectively $\left(\mathrm{Neuro}^{\mathrm{CTR}}+\mathrm{Ast}^{\mathrm{CTR}}\right.$ : $0.047 \pm 0.004$, Neuro $^{\mathrm{HD}}+\mathrm{Ast}^{\mathrm{CTR}}: \quad 0.060 \pm 0.006, \quad \mathrm{Neuro}^{\mathrm{CTR}}+\mathrm{Ast}^{\mathrm{HD}}$ : $0.094 \pm 0.007$, Neuro ${ }^{\mathrm{HD}}+\mathrm{Ast}^{\mathrm{HD}}: 0.092 \pm 0.007$ absorbance; $p<0.005$ vs. control neuron; $p<0.001$ vs HD neuron; Figure 4K). Collectively, this data indicates that the $\mathrm{HD}$ astrocytes are less proficient in preventing glutamate-induced toxicity in neuron co-cultures compared to control astrocytes, regardless of the disease state of the neurons.

\section{GABA Expression Is Reduced in Neurons Co-cultured With HD Astrocytes Compared to Control Astrocytes}

An interesting observation of cells in mono-culture was that astrocytes had significantly larger nuclei $(p<0.0001$; Figure 5A) compared to the smaller nuclei (Figure 5B) in iPSC-derived neurons, which was consistent in co-cultures $(p<0.0001$; Supplementary Figure 4). This characteristic was used to subsequently discriminate between astrocytes and neurons. Additionally, both HD astrocytes (CTR Ast: $361.7 \pm 24.93$, HD

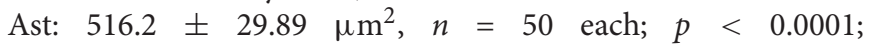
Figure 5) and neurons (CTR Neuro: 68.18 \pm 2.22, HD

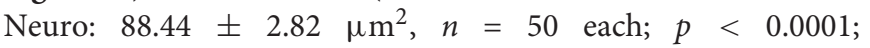
Figure 5B) had significantly larger nuclei compared to the respective control cells.

The neurotransmitter GABA is traditionally a marker of inhibitory neurons (Molgaard et al., 2014), but it is also expressed in glia (Lee et al., 2011), and levels of expression are brain-region dependent (Yoon et al., 2011). Therefore, we first assessed whether GABA expression differed in HD and control lines. Immunocytochemistry showed that the cellular localization of GABA was completely different in neurons (small nuclei; Figures 5C,E) and astrocytes (large nuclei; Figures 5D,E). IPSC-derived neurons had classical cytoplasmic GABA expression in the form of dim puntca throughout the cytoplasm, whereas astrocytes mainly expressed GABA in bright perinuclear foci (Jo et al., 2014). We found that more HD astrocytes expressed GABA-positive perinuclear foci than control astrocytes, and this pattern was independent of culture state $\left(\mathrm{Ast}^{\mathrm{CTR}}\right.$ : $11.86 \pm 3.50, \mathrm{Ast}^{\mathrm{HD}}$ : $29.72 \pm 4.66$, Ast $^{\text {CTR }}+$ Neuro ${ }^{\text {CTR }} 10.56 \pm 2.55$, Ast $^{\text {CTR }}+$ Neuro $^{\text {HD }} 12.23 \pm 1.45$, $\mathrm{Ast}^{\mathrm{HD}}+\mathrm{Neuro}^{\mathrm{CTR}} 27.80 \pm 2.69$. Ast $^{\mathrm{HD}}+\mathrm{Neuro}^{\mathrm{HD}} 25.59 \pm 4.38$ bright GABA+ cells of total, $n=50$ cells each, three experiments; Figure 5F). Interestingly, while there was no difference in the percentage of $\mathrm{HD}$ or control GABA-positive neurons in mono-culture, the percentage of GABA-positive control neurons increased when co-cultured with control astrocytes (Neuro ${ }^{\mathrm{CTR}}$ : $44.63 \pm 2.03$, Neuro ${ }^{\mathrm{HD}}$ : $33.44 \pm 1.53$, Ast $^{\text {CTR }}+$ Neuro $^{\text {CTR }} 67.98 \pm 0.38$, Ast ${ }^{\text {CTR }}+$ Neuro $^{\text {HD }} 39.35 \pm 5.25$, $\mathrm{Ast}^{\mathrm{HD}}+\mathrm{Neuro}^{\mathrm{CTR}} 38.94 \pm 2.43$, Ast $^{\mathrm{HD}}+\mathrm{Neuro}^{\mathrm{HD}} 36.25 \pm 1.42$ $\operatorname{dim}$ GABA + cells of total, $n=50$ cells each, three experiments; Figure 5G). This supports reports that astrocyte co-culture is beneficial for neurogenesis and functional maturation (Johnson et al., 2007). However, this astrocyte-facilitated augmentation did not occur when HD astrocytes were cultured with either control or HD neurons, indicating that HD astrocytes are not as supportive of neuronal maturation as control astrocytes. Additionally, control neurons co-cultured with control astrocytes demonstrated increased GABA expression, but this increase was not observed in HD neurons, suggesting an inherent deficiency in HD neurons that cannot be rescued by control glia.

\section{DISCUSSION}

Here, we present data from a novel protocol to generate iPSC-derived astrocytes that are physiologically mature. Using this protocol, HD astrocytes recapitulate phenotypes previously seen in HD mouse models, such as impairment of $\mathrm{K}_{i r}$ currents, lengthened spontaneous $\mathrm{Ca}^{2+}$ waves, and reduced cell membrane capacitance. They also demonstrated that while the control astrocytes lead to increased neuronal maturation, HD astrocytes were poorly supportive of neuronal functional maturation. Overall, this study supports and extends the idea that there are non-cell autonomous 
A
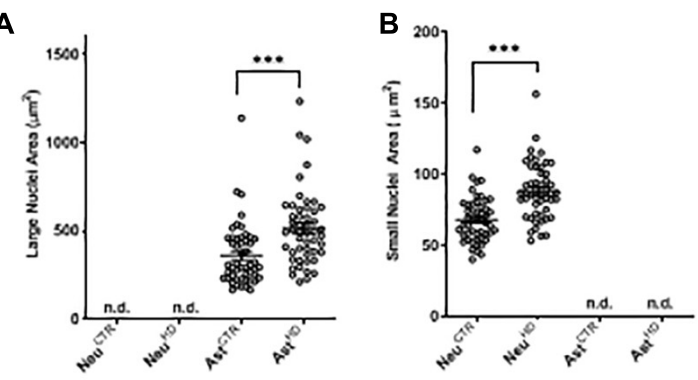

E

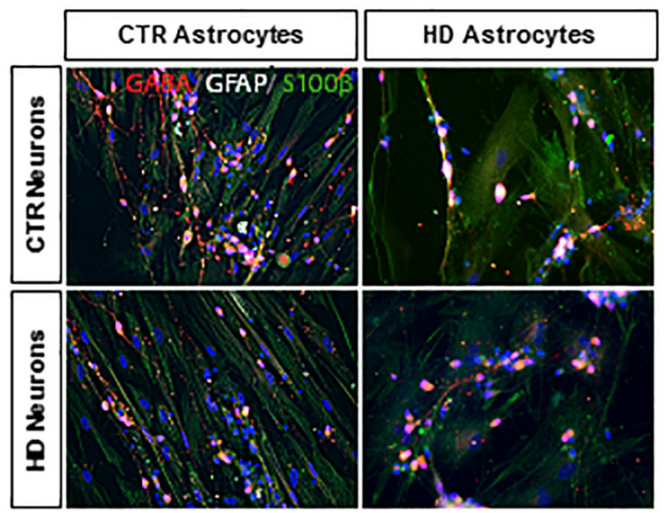

C

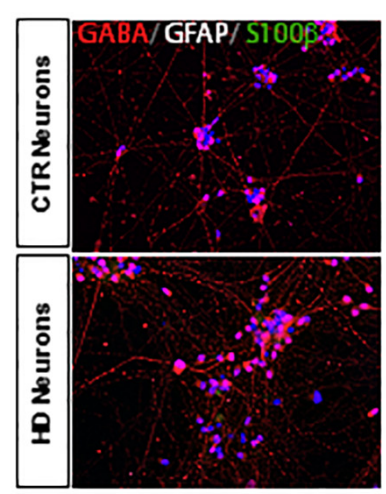

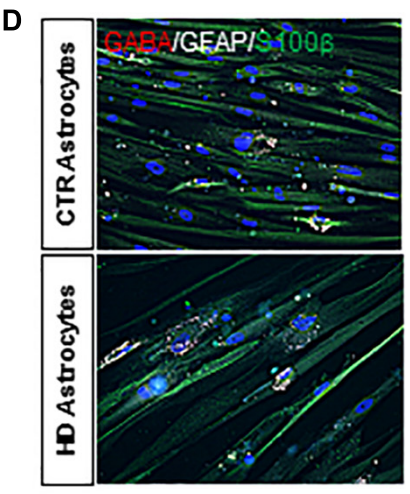

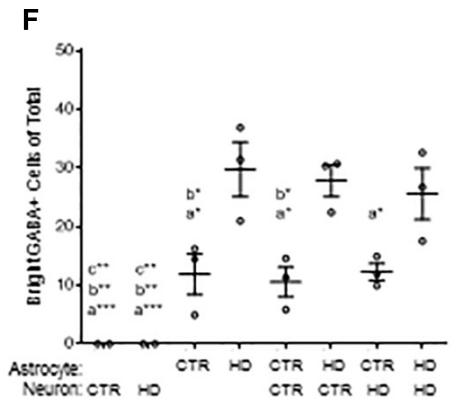

G

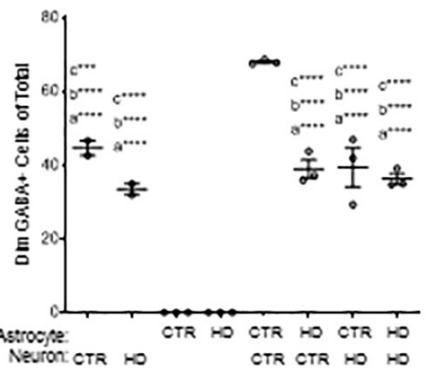

FIGURE 5 | Stage 4 HD (HD109) and control (CTR33) astrocytes (large nuclei, > $210 \mu \mathrm{m}^{2}$ ) and neurons (small nuclei, <141 $\mu \mathrm{m}^{2}$ ) in mono- or co-culture display differences in both nuclear size and percent of cells expressing GABA. (A) HD astrocyte nuclei are larger than control astrocytes. No large nuclei are detected in the neuronal cultures. (B) HD neuronal nuclei are larger than control neuronal nuclei. No small nuclei are detected in the Stage 4 astrocyte cultures. (C-E) GABA (red), GFAP (white) and S100ß (green) expression in panel (C) control and HD neurons, (D) control and HD astrocytes, (E) co-culture matrix of HD and control astrocytes/neurons. (F) HD astrocytes in mono-culture or co-culture have a higher percentage of cells expressing a dim, punctate GABA compared to control astrocytes. Significance between conditions denoted as "a": from Ast ${ }^{H D}$, "b": from NeuCTR + Ast ${ }^{H D}$, "c": from Ast ${ }^{H D}+$ Neu ${ }^{H D}$. (G) Control neurons in mono-culture have a higher percentage of cells expressing a bright cytoplasmic GABA stain than HD neurons in mono-culture, which is increased upon addition of control astrocyte co-culture. Significance between conditions denoted as "a": from Ast ${ }^{\text {CTR , "b": from Ast }}$ "HD, "c": from Ast ${ }^{\text {CTR }}+$ NeuCTR . These cells were analyzed from more than three separate experiments. Ast, astrocyte; Neu, neuron; CTR, control. One-way ANOVA $\left({ }^{*} p<0.05,{ }^{* *} p<0.01,{ }^{* * *} p<0.001\right.$, and $\left.{ }^{* * * *} p<0.0001\right)$.

contributions of astrocytes to the HD phenotype and shows that iPSC-derived astrocytes and neurons in co-culture can be used to effectively model HD.

\section{Electrophysiological Assessment of iPSC-Derived Astrocytes in vitro}

Maturation of our astrocyte cultures was examined via electrophysiological assessment. As expected, the intrinsic membrane properties differed between immature (Type A and B) and mature (Type C and D) astrocytes, which validated a specification of maturation states (Higashi et al., 2001; Kofuji and Newman, 2004). As evident in primary rodent astrocytes, specific differences in intrinsic properties are observed in electrophysiologicaly distinct astrocyte populations of which the proportion of populations present changes as the animal ages (Kimelberg et al., 2000; Zhou et al., 2006). For example, astrocytes of the newborn rat hippocampus exhibit high membrane resistance and depolarized membrane potentials, which decrease and hyperpolarize with age, respectively. We observed a similar trend in properties measured in our iPSC-derived astrocyte Types. The astrocyte Types of our cultures were further validated by expression of specific
$\mathrm{K}^{+}$currents that are expressed by astrocytes in vivo across development. In the rat hippocampus, the most prevalent population of astrocytes in young animals (P1-P15) exhibit outward-rectifying, voltage-gated potassium currents, whereas the more mature astrocyte population observed in older animals (P8-P106) presented more ohmic features of passive potassium currents (Zhou et al., 2006). However, it is important to note that our mature Type D astrocytes reach a maturation profile comparable to intermediately mature astrocytes observed in rodent preparations. This is not surprising, as human iPSCderived models have been shown to achieve maturation levels similar to that observed in human fetal tissue yet fall short of adult tissues based upon transcriptomic analysis (Ho et al., 2016). Still, the trend identified in our astrocyte Types that show maturation of intrinsic properties and expression of specific potassium currents indicate that regardless of disease state, Type $\mathrm{C} / \mathrm{D}$ astrocytes were the most mature astrocytes with generally larger and more complex physiology.

\section{Glial Dysfunction in HD}

Reactive astro-gliosis can be identified as an early feature of $\mathrm{HD}$, due to the increased GFAP immunoreactivity in 
prodromal gene-positive patients that continues to increase with disease progression (Faideau et al., 2010). HD patient iPSCderived astrocytes display autophagocytic vacuoles (Juopperi et al., 2012) and increased evoked inflammatory responses (Hsiao et al., 2014). In our study, the iPSC-derived HD astrocytes were found to have larger cell capacitances than control astrocytes, indicative of an increased cell size. This increase in astrocyte cell size is linked to reactive astrocytosis (Amenta et al., 1998). Additionally, larger nuclei have been previously observed in HD astrocytes, which has been attributed to a reactive astrocyte pathotype (Aschner and Kimelberg, 1996). This mirrors the larger HD astrocyte nuclei that was also observed in our study. Additionally, increased GABA expression observed in $\mathrm{HD}$ astrocytes has been linked to reactive astrocytosis (Jo et al., 2014), and, depolarized HD astrocytes have a reduced ability to release GABA via GAT3 (Wójtowicz et al., 2013). This could explain the accumulation of GABA seen in HD astrocytes in our study. Another explanation could be a reduced capability for $\mathrm{HD}$ astrocytes to properly catabolize GABA. It has been previously demonstrated that in $\mathrm{HD}$ animal astrocytes GABA-glutamine cycling is compromised (Skotte et al., 2018), and this could lead to an astrocytic accumulation of GABA. Together, these observations truly suggest that in vitro generation of $\mathrm{HD}$ iPSC-derived mature astrocytes generates a reactive astrocyte population similarly to that observed in vivo.

Astrocytes in HD mouse models have alterations in mechanisms involved in extracellular ionic homeostasis, including a decrease in expression of astrocyte-enriched $\mathrm{K}_{i r} 4.1$ channels (Tong et al., 2014). This reduction in $\mathrm{K}_{i r}$ current contributes to dysfunctional astrocyte glutamate and $\mathrm{Ca}^{2+}$ signaling at a stage in mouse models which is similar to the prodromal stage in patients (Jiang et al., 2016). This dyshomeostasis of astrocytes contributes to the neuronal loss seen in disease, due to elevated extracellular $\mathrm{K}^{+}$and the concomitant augmentation of neuronal excitability linked to neuronal toxicity in HD (Tong et al., 2014). In fact, overexpression of $\mathrm{K}_{i r} 4.1$ channels in $\mathrm{HD}$ mouse astrocytes in vivo normalizes extracellular $\mathrm{K}^{+}$, reverses aspects of neuronal dysfunction, increases survival and attenuates some motor phenotypes. In the present study, the HD iPSC-derived Type $\mathrm{C}$ astrocytes displayed reduced peak $\mathrm{K}_{i r}$ current densities compared to control astrocytes, similar to the reduction seen in vivo (Tong et al., 2014). This $\mathrm{K}_{\text {ir }}$ current density reduction was not mirrored in either the Type $\mathrm{A} / \mathrm{B} \quad \mathrm{K}_{v}$ or Type $\mathrm{D} \mathrm{K}_{2 P}$ current densities. It has been previously demonstrated that immature glial cells lack expression of $\mathrm{K}_{i r}$ channels (Sontheimer, 1994; Bordey and Sontheimer, 1997; MacFarlane and Sontheimer, 2000), which would explain the lack of phenotype in the Type A/B population. Upon astrocyte maturation, an increase in $\mathrm{K}_{i r}$ channel expression causes a hyperpolarization of RMP from -50 to $-80 \mathrm{mV}$ (Ransom and Sontheimer, 1995; Bordey and Sontheimer, 1997). This hyperpolarization is mirrored in the Type A/B transition to Type C/D astrocytes. Not surprisingly then, the depolarized resting membrane potential in Type C HD astrocytes coincides with the reduced $\mathrm{K}_{i r}$ current density.
Similarly, the absence of a significant difference between $\mathrm{HD}$ and control astrocytes in $\mathrm{K}^{+}$current density was consistent with a lack of difference in RMP in the Type D astrocytes, which display passive $\mathrm{K}_{2 P}$ currents rather than the $\mathrm{K}_{\text {ir }}$ currents.

\section{Non-cell Autonomous Effects Seen in HD Using Glial-Neuronal Co-culture Paradigms}

Non-cell autonomous effects of astrocytes in $\mathrm{HD}$ have been demonstrated. Control murine neurons co-cultured with HD glia are more susceptible to glutamate excitotoxicity (Shin et al., 2005). Conversely, control murine glia provide protection from excitotoxicity as seen in primary HD mouse neurons (Shin et al., 2005). The data presented here with human iPSC-derived astrocyte-neuron co-culture recapitulate those findings. When we co-cultured control iPSC-derived neurons with control iPSC-derived astrocytes, the neurons had an increased proportion of GABA-expressing cells and an increase in spontaneous activity at early stages of co-culture. These are indicative that control astrocytes support increased neuronal maturation more efficiently than neuronal mono-culture. This is not surprising, as the benefit of astrocyte co-culture for neuronal maturation and support is well established (Johnson et al., 2007). However, when control neurons were plated onto HD astrocytes for 6 days, there was no increase in neuronal GABA expression, and while there was increased spontaneous activity as compared to in mono-culture, it was markedly less than co-culture with control astrocytes. In agreement with the delayed development phenotype previously proposed for HD iPSCderived models (Mattis et al., 2015; Ring et al., 2015; HD iPSC Consortium, 2017), the HD and control neurons co-cultured with $\mathrm{HD}$ astrocytes did achieve similar firing rates after extended time in co-culture. Additionally, cocultures of either control or HD neurons with HD astrocytes demonstrated increased toxicity after chronic glutamate exposure, as compared to neurons on control astrocytes. These observations demonstrate that the $\mathrm{HD}$ astrocytes do not support neuronal maturation or function as effectively as control astrocytes. This agrees with previous data demonstrating that HD murine glia have a reduced ability to support neurons due to a reduced ability to remove extracellular glutamate, which contributes to the neuronal excitotoxic pathotype in HD (Shin et al., 2005).

In this study, we generated physiologically mature iPSCderived astrocytes from HD patients and control subjects. More excitingly, these astrocytes recapitulated an important astrocyte HD pathology previously measured in mouse models, the impairment of $\mathrm{K}_{i r}$ currents, which had not yet been measured in humans. Furthermore, we employed this novel, iPSC-based human cell model to identify additional astrocyte $\mathrm{HD}$-pathotypes, including lengthened spontaneous $\mathrm{Ca}^{2+}$ waves and reduced cell membrane capacitance. In the future, this human $\mathrm{HD}$ patient iPSC-based neuronal-glial co-culture system can be used to recapitulate key aspects of 
the disease for further elucidation of mechanistic pathways and therapeutic intervention.

\section{DATA AVAILABILITY}

All datasets generated for this study are included in the manuscript and/or the Supplementary Files.

\section{ETHICS STATEMENT}

All procedures were performed in accordance with the IRB guidelines at the Cedars-Sinai Medical Center under the auspice IRB-SCRO Protocols: Pro00028429, Pro00021505, and Pro00032834.

\section{AUTHOR CONTRIBUTIONS}

CS, PK, NA, VG, DR, and VM designed the study. DR, VG, and CT carried out the study. DR, VG, and VM analyzed the data. VG and VM wrote the manuscript.

\section{REFERENCES}

Amenta, F., Bronzetti, E., Sabbatini, M., and Vega, J. A. (1998). Astrocyte changes in aging cerebral cortex and hippocampus: a quantitative immunohistochemical study. Microsc. Res. Tech. 43, 29-33. doi: 10.1002/(sici)1097-0029(19981001)43:1<29::aid-jemt5>3.0.co;2-h

Andrew, S. E., Goldberg, Y. P., Kremer, B., Telenius, H., Theilmann, J., Adam, S., et al. (1993). The relationship between trinucleotide (CAG) repeat length. Nat. Genet. 4, 398-403. doi: 10.1038/ng0893-398

Aschner, M., and Kimelberg, H. K. (1996). The Role of Glia in Neurotoxicity. Boca Raton,FL: CRC Press.

Benraiss, A., Wang, S., Herrlinger, S., Li, X., Chandler-Militello, D., Mauceri, J., et al. (2016). Human glia can both induce and rescue aspects of disease phenotype in Huntington disease. Nat. Commun. 7:11758. doi: 10.1038/ ncomms 11758

Bordey, A., and Sontheimer, H. (1997). Postnatal development of ionic currents in rat hippocampal astrocytes in situ. J. Neurophysiol. 78, 461-477. doi: 10.1152/ jn.1997.78.1.461

Bradford, J., Shin, J. Y., Roberts, M., Wang, C. E., Li, X. J., and Li, S. (2009). Expression of mutant huntingtin in mouse brain astrocytes causes age-dependent neurological symptoms. Proc. Natl. Acad Sci. U.S.A. 106, 22480-22485. doi: 10.1073/pnas.0911503106

Chung, W. S., Allen, N. J., and Eroglu, C. (2015). Astrocytes control synapse formation, function, and elimination. Cold Spring Harb. Perspect. Biol. 7:a020370. doi: 10.1101/cshperspect.a020370

Ebert, A. D., Shelley, B. C., Hurley, A. M., Onorati, M., Castiglioni, V., Patitucci, T. N., et al. (2013). EZ spheres: a stable and expandable culture system for the generation of pre-rosette multipotent stem cells from human ESCs and iPSCs. Stem Cell Res. 10, 417-427. doi: 10.1016/j.scr.2013.01.009

Ebert, A. D., Yu, J., Rose, F. F., Mattis, V. B., Lorson, C. L., Thomson, J. A., et al. (2009). Induced pluripotent stem cells from a spinal muscular atrophy patient. Nature 457, 277-280. doi: 10.1038/nature07677

Faideau, M., Kim, J., Cormier, K., Gilmore, R., Welch, M., Auregan, G., et al. (2010). In vivo expression of polyglutamine-expanded huntingtin by mouse striatal astrocytes impairs glutamate transport: a correlation with Huntington's disease subjects. Hum. Mol. Genet. 19, 3053-3067. doi: 10.1093/hmg/ddq212

HD iPSC Consortium (2012). Induced pluripotent stem cells from patients with Huntington's disease show CAG-repeat-expansion-associated phenotypes. Cell Stem Cell 11, 264-278. doi: 10.1016/j.stem.2012.04.027

\section{FUNDING}

This work was partially funded by an MRC VIP Award to DR, NA, and PK, and funded by the Board of Governors Regenerative Medicine Institute at the Cedars-Sinai Medical Center.

\section{ACKNOWLEDGMENTS}

We thank Berhan Mandefro and Brandon Shelley for cell maintenance and the iPSC Core Facility in the Board of Governors Regenerative Medicine Institute for generation and characterization of the iPSC lines. We also thank Dr. Soshana Svendsen for assistance in writing the manuscript.

\section{SUPPLEMENTARY MATERIAL}

The Supplementary Material for this article can be found online at: https://www.frontiersin.org/articles/10.3389/fnins. 2019.00669/full\#supplementary-material

HD iPSC Consortium (2017). Developmental alterations in Huntington's disease neural cells and pharmacological rescue in cells and mice. Nat. Neurosci. 20, 648-660. doi: 10.1038/nn.4532

Higashi, K., Fujita, A., Inanobe, A., Tanemoto, M., Doi, K., Kubo, T., et al. (2001). An inwardly rectifying $\mathrm{K}(+)$ channel, Kir4.1, expressed in astrocytes surrounds synapses and blood vessels in brain. Am. J. Physiol. Cell Physiol. 281, C922-C931. doi: 10.1152/ajpcell.2001.281.3.C922

Ho, R., Sances, S., Gowing, G., Amoroso, M. W., O’Rourke, J. G., Sahabian, A., et al. (2016). ALS disrupts spinal motor neuron maturation and aging pathways within gene co-expression networks. Nat. Neurosci. 19, 1256-1267. doi: $10.1038 / \mathrm{nn} .4345$

Hsiao, H. Y., Chiu, F. L., Chen, C. M., Wu, Y. R., Chen, H. M., Chen, Y. C., et al. (2014). Inhibition of soluble tumor necrosis factor is therapeutic in Huntington's disease. Hum. Mol. Genet. 23, 4328-4344. doi: 10.1093/hmg/ ddu151

Jiang, R., Diaz-Castro, B., Looger, L. L., and Khakh, B. S. (2016). Dysfunctional calcium and glutamate signaling in striatal astrocytes from Huntington's disease model mice. J. Neurosci. 36, 3453-3470. doi: 10.1523/JNEUROSCI.3693-15. 2016

Jimenez-Sanchez, M., Licitra, F., Underwood, B. R., and Rubinsztein, D. C. (2017). Huntington's Disease: mechanisms of pathogenesis and therapeutic strategies. Cold Spring Harb. Perspect. Med. 7:a024240. doi: 10.1101/cshperspect. a024240

Jo, S., Yarishkin, O., Hwang, Y. J., Chun, Y. E., Park, M., Woo, D. H., et al. (2014). GABA from reactive astrocytes impairs memory in mouse models of Alzheimer's disease. Nat. Med. 20, 886-896. doi: 10.1038/nm. 3639

Johnson, M. A., Weick, J. P., Pearce, R. A., and Zhang, S. C. (2007). Functional neural development from human embryonic stem cells: accelerated synaptic activity via astrocyte coculture. J. Neurosci. 27, 3069-3077. doi: 10.1523/ JNEUROSCI.4562-06.2007

Juopperi, T. A., Kim, W. R., Chiang, C.-H., Yu, H., Margolis, R. L., Ross, C. A., et al. (2012). Astrocytes generated from patient induced pluripotent stem cells recapitulate features of Huntington's disease patient cells. Mol. brain 5:17. doi: 10.1186/1756-6606-5-17

Kemp, P. J., Rushton, D. J., Yarova, P. L., Schnell, C., Geater, C., Hancock, J. M., et al. (2016). Improving and accelerating the differentiation and functional maturation of human stem cell-derived neurons: role of extracellular calcium and GABA. J. Physiol. 594, 6583-6594. doi: 10.1113/JP270655 
Khakh, B. S., Beaumont, V., Cachope, R., Munoz-Sanjuan, I., Goldman, S. A., and Grantyn, R. (2017). Unravelling and exploiting astrocyte dysfunction in Huntington's disease. Trends Neurosci. 40, 422-437. doi: 10.1016/j.tins.2017. 05.002

Khakh, B. S., and Sofroniew, M. V. (2014). Astrocytes and Huntington's disease. ACS Chem. Neurosci. 5, 494-496. doi: 10.1021/cn500100r

Kimelberg, H. K., Schools, G. P., Cai, Z., and Zhou, M. (2000). Freshly isolated astrocyte (FIA) preparations: a useful single cell system for studying astrocyte properties. J. Neurosci. Res. 61, 577-587. doi: 10.1002/1097-4547(20000915)61: $6<577:$ :aid-jnr1 $>3.0$. co; $2-\mathrm{t}$

Kofuji, P., and Newman, E. A. (2004). Potassium buffering in the central nervous system. Neuroscience 129, 1045-1056. doi: 10.1016/j.neuroscience.2004.06.008

Lai, B., Mao, X. O., Xie, L., Chang, S. Y., Xiong, Z. G., Jin, K., et al. (2010). Electrophysiological properties of subventricular zone cells in adult mouse brain. Brain Res. 1340, 96-105. doi: 10.1016/j.brainres.2010.04.057

Lee, M., Schwab, C., and McGeer, P. L. (2011). Astrocytes are GABAergic cells that modulate microglial activity. Glia 59, 152-165. doi: 10.1002/glia.21087

Lim, D., Fedrizzi, L., Tartari, M., Zuccato, C., Cattaneo, E., Brini, M., et al. (2008). Calcium homeostasis and mitochondrial dysfunction in striatal neurons of Huntington disease. J. Biol. Chem. 283, 5780-5789. doi: 10.1074/jbc. M704704200

Macdonald, V., and Halliday, G. (2002). Pyramidal cell loss in motor cortices in Huntington's disease. Neurobiol. Dis. 10, 378-386. doi: 10.1006/nbdi.2002.0528

MacFarlane, S. N., and Sontheimer, H. (2000). Modulation of Kv1.5 currents by src tyrosine phosphorylation: potential role in the differentiation of astrocytes. J. Neurosci. 20, 5245-5253. doi: 10.1523/jneurosci.20-14-05245.2000

Mattis, V. B., Tom, C., Akimov, S., Saeedian, J., Østergaard, M. E., Southwell, A. L., et al. (2015). HD iPSC-derived neural progenitors accumulate in culture and are susceptible to BDNF withdrawal due to glutamate toxicity. Hum. Mol. Genet. 24, 3257-3271. doi: 10.1093/hmg/ddv080

McKhann, G. M., D’Ambrosio, R., and Janigro, D. (1997). Heterogeneity of astrocyte resting membrane potentials and intercellular coupling revealed by whole-cell and gramicidin-perforated patch recordings from cultured neocortical and hippocampal slice astrocytes. J. Neurosci. 17, 6850-6863. doi: 10.1523/jneurosci.17-18-06850.1997

Milnerwood, A. J., Gladding, C. M., Pouladi, M. A., Kaufman, A. M., Hines, R. M., Boyd, J. D., et al. (2010). Early increase in extrasynaptic NMDA receptor signaling and expression contributes to phenotype onset in Huntington's disease mice. Neuron 65, 178-190. doi: 10.1016/j.neuron.2010.01.008

Molgaard, S., Ulrichsen, M., Boggild, S., Holm, M. L., Vaegter, C., Nyengaard, J., et al. (2014). Immunofluorescent visualization of mouse interneuron subtypes. F1000Res. 3:242. doi: 10.12688/f1000research.5349.2

Nelson, A. D., Suzuki, M., and Svendsen, C. N. (2008). A high concentration of epidermal growth factor increases the growth and survival of neurogenic radial glial cells within human neurosphere cultures. Stem Cells 26, 348-355. doi: 10.1634/stemcells.2007-0299

Pfrieger, F. W. (2010). Role of glial cells in the formation and maintenance of synapses. Brain Res. Rev. 63, 39-46. doi: 10.1016/j.brainresrev.2009.11.002

Pfrieger, F. W., and Barres, B. A. (1997). Synaptic efficacy enhanced by glial cells in vitro. Science 277, 1684-1687. doi: 10.1126/science.277.5332. 1684

Ransom, C. B., and Sontheimer, H. (1995). Biophysical and pharmacological characterization of inwardly rectifying $\mathrm{K}+$ currents in rat spinal cord astrocytes. J. Neurophysiol. 73, 333-346. doi: 10.1152/jn.1995.73.1.333

Raponi, E., Agenes, F., Delphin, C., Assard, N., Baudier, J., Legraverend, C., et al. (2007). S100B expression defines a state in which GFAP-expressing cells lose their neural stem cell potential and acquire a more mature developmental stage. Glia 55, 165-177. doi: 10.1002/glia.20445

Ring, K. L., An, M. C., Zhang, N., O’Brien, R. N., Ramos, E. M., Gao, F., et al. (2015). genomic analysis reveals disruption of striatal neuronal development and therapeutic targets in human Huntington's disease neural stem cells. Stem Cell Rep. 5, 1023-1038. doi: 10.1016/j.stemcr.2015.11.005

Ross, C. A., and Tabrizi, S. J. (2011). Huntington's disease: from molecular pathogenesis to clinical treatment. Lancet Neurol 10, 83-98. doi: 10.1016/ S1474-4422(10)70245-3

Seifert, G., Hüttmann, K., Binder, D. K., Hartmann, C., Wyczynski, A., Neusch, C., et al. (2009). Analysis of astroglial K+ channel expression in the developing hippocampus reveals a predominant role of the Kir4.1 subunit. J. Neurosci. 29, 7474-7488. doi: 10.1523/JNEUROSCI.3790-08. 2009

Shelton, M. K., and McCarthy, K. D. (1999). Mature hippocampal astrocytes exhibit functional metabotropic and ionotropic glutamate receptors in situ. Glia 26, 1-11. doi: 10.1002/(sici)1098-1136(199903)26:1<1::aid-glia1>3.0.co;2-Z

Shin, J. Y., Fang, Z. H., Yu, Z. X., Wang, C. E., Li, S. H., and Li, X. J. (2005). Expression of mutant huntingtin in glial cells contributes to neuronal excitotoxicity. J. Cell Biol. 171, 1001-1012. doi: 10.1083/jcb.200508072

Skotte, N. H., Andersen, J. V., Santos, A., Aldana, B. I., Willert, C. W., Nørremølle, A., et al. (2018). Integrative characterization of the R6/2 mouse model of huntington's disease reveals dysfunctional astrocyte metabolism. Cell Rep. 23, 2211-2224. doi: 10.1016/j.celrep.2018.04.052

Sontheimer, H. (1994). Voltage-dependent ion channels in glial cells. Glia 11, 156-172. doi: 10.1002/glia.440110210

Spira, M. E., and Hai, A. (2013). Multi-electrode array technologies for neuroscience and cardiology. Nat. Nanotechnol. 8, 83-94. doi: 10.1038/nnano. 2012.265

Takahashi, K., and Yamanaka, S. (2006). Induction of pluripotent stem cells from mouse embryonic and adult fibroblast cultures by defined factors. Cell 126, 663-676. doi: 10.1016/j.cell.2006.07.024

Tang, X., Zhou, L., Wagner, A. M., Marchetto, M. C., Muotri, A. R., Gage, F. H., et al. (2013). Astroglial cells regulate the developmental timeline of human neurons differentiated from induced pluripotent stem cells. Stem Cell Res. 11, 743-757. doi: 10.1016/j.scr.2013.05.002

Telezhkin, V., Schnell, C., Yarova, P., Yung, S., Cope, E., Hughes, A., et al. (2016). Forced cell cycle exit and modulation of GABAA, CREB, and GSK3 $\beta$ signaling promote functional maturation of induced pluripotent stem cellderived neurons. Am. J. Physiol. Cell Physiol. 310, C520-C541. doi: 10.1152/ ajpcell.00166.2015

Thu, D. C., Oorschot, D. E., Tippett, L. J., Nana, A. L., Hogg, V. M., Synek, B. J., et al. (2010). Cell loss in the motor and cingulate cortex correlates with symptomatology in Huntington's disease. Brain 133(Pt 4), 1094-1110. doi: 10. 1093/brain/awq047

Tong, X., Ao, Y., Faas, G. C., Nwaobi, S. E., Xu, J., Haustein, M. D., et al. (2014). Astrocyte Kir4.1 ion channel deficits contribute to neuronal dysfunction in Huntington's disease model mice. Nat. Neurosci. 17, 694-703. doi: 10.1038/nn. 3691

Wójtowicz, A. M., Dvorzhak, A., Semtner, M., and Grantyn, R. (2013). Reduced tonic inhibition in striatal output neurons from Huntington mice due to loss of astrocytic GABA release through GAT-3. Front. Neural. Circuits 7:188. doi: 10.3389/fncir.2013.00188

Wright, L. S., Li, J., Caldwell, M. A., Wallace, K., Johnson, J. A., and Svendsen, C. N. (2003). Gene expression in human neural stem cells: effects of leukemia inhibitory factor. J. Neurochem. 86, 179-195. doi: 10.1046/j.1471-4159.2003. 01826.x

Yoon, B. E., Jo, S., Woo, J., Lee, J. H., Kim, T., Kim, D., et al. (2011). The amount of astrocytic GABA positively correlates with the degree of tonic inhibition in hippocampal CA1 and cerebellum. Mol. Brain 4:42. doi: 10.1186/1756-660 6-4-42

Zhong, S., Du, Y., Kiyoshi, C. M., Ma, B., Alford, C. C., Wang, Q., et al. (2016). Electrophysiological behavior of neonatal astrocytes in hippocampal stratum radiatum. Mol. Brain 9:34. doi: 10.1186/s13041-016-0213-7

Zhou, M., Schools, G. P., and Kimelberg, H. K. (2006). Development of GLAST(+) astrocytes and $\mathrm{NG} 2(+)$ glia in rat hippocampus CA1: mature astrocytes are electrophysiologically passive. J. Neurophysiol. 95, 134-143. doi: 10.1152/jn. 00570.2005

Conflict of Interest Statement: The authors declare that the research was conducted in the absence of any commercial or financial relationships that could be construed as a potential conflict of interest.

Copyright (C) 2019 Garcia, Rushton, Tom, Allen, Kemp, Svendsen and Mattis. This is an open-access article distributed under the terms of the Creative Commons Attribution License (CC BY). The use, distribution or reproduction in other forums is permitted, provided the original author(s) and the copyright owner(s) are credited and that the original publication in this journal is cited, in accordance with accepted academic practice. No use, distribution or reproduction is permitted which does not comply with these terms. 